\title{
Effect of Seasoning Addition on Volatile Composition and Sensory Properties of Stewed Pork
}

\author{
Dong Han ${ }^{1,2}$, Chun-Hui Zhang ${ }^{1, *}$ and Marie-Laure Fauconnier ${ }^{2} \mathbb{D}$ \\ 1 Laboratory of Agro-Products Processing, Ministry of Agriculture and Rural Affairs, \\ Institute of Food Science and Technology, Chinese Academy of Agricultural Sciences, Beijing 100193, China; \\ orange_1101@126.com \\ 2 Laboratory of Chemistry of Natural Molecules, Gembloux Agro-Bio Technology, University of Liege, \\ 5030 Gembloux, Belgium; marie-laure.fauconnier@uliege.be \\ * Correspondence: dr_zch@163.com; Tel.: +86-10-62819469
}

check for updates

Citation: Han, D.; Zhang, C.-H.; Fauconnier, M.-L. Effect of Seasoning Addition on Volatile Composition and Sensory Properties of Stewed Pork. Foods 2021, 10, 83. https:// doi.org/10.3390/foods10010083

Received: 1 December 2020 Accepted: 30 December 2020 Published: 4 January 2021

Publisher's Note: MDPI stays neutral with regard to jurisdictional clai$\mathrm{ms}$ in published maps and institutional affiliations.

Copyright: () 2021 by the authors. Licensee MDPI, Basel, Switzerland. This article is an open access article distributed under the terms and conditions of the Creative Commons Attribution (CC BY) license (https:// creativecommons.org/licenses/by/ $4.0 /)$.
Abstract: The study aimed to investigate the influence of seasoning formulations $\left(\mathrm{SP}_{1}\right.$ : water; $\mathrm{SP}_{2}$ : water and salt; $\mathrm{SP}_{3}$ : water, salt and spices; $\mathrm{SP}_{4}$ : water, salt, spices and soy sauce; $\mathrm{SP}_{5}$ : water, salt, spices, soy sauce, sugar; $\mathrm{SP}_{6}$ : water, salt, spices, soy sauce, sugar and cooking wine) on the volatile profiles and sensory evaluation of stewed pork. Volatile compounds were extracted using solid phase microextraction (SPME), then analysed by gas chromatography-mass spectrometry/olfactometry (GC$\mathrm{MS} / \mathrm{O}$ ) and two-dimensional gas chromatographic combined with time-of-fight mass spectrometry $(\mathrm{GC} \times \mathrm{GC}-\mathrm{TOFMS})$. The results revealed that the most abundant volatile compounds, especially aldehydes, were presented in the stewed pork using $\mathrm{SP}_{1}$ and $\mathrm{SP}_{2}$. This indicated that the stewed pork with water and salt could promote lipid oxidation and amino acid degradation. As revealed by principal component analysis (PCA), the stewed pork samples with $\mathrm{SP}_{3}$ were located on the opposite side of that with $\mathrm{SP}_{4}, \mathrm{SP}_{5}$, and $\mathrm{SP}_{6}$ in the first and third principal component (PC1-PC3), which indicated that the overall flavour formed by adding spices was significantly different from that of adding soy sauce, sugar, and cooking wine. Sensory evaluation showed that stronger spicy, caramel, and soy sauce odour were present in samples $\mathrm{SP}_{3}, \mathrm{SP}_{4}, \mathrm{SP}_{5}$, and $\mathrm{SP}_{6}$. This study has indicated that the addition of food seasoning had a positive effect on flavour profiles of stewed pork, particularly for salt and spices.

Keywords: stewed pork; seasoning formulations; volatile components; gas chromatography-mass spectrometry/olfactometry (GC-MS/O); two-dimensional gas chromatographic combined with time-of-fight mass spectrometry (GC $\times$ GC-TOFMS); principal component analysis (PCA)

\section{Introduction}

According to the report of State Statistical Bureau of China, pork production has increased to 54.0 million tons from 2014 to 2019 , accounting for more than $60 \%$ of the meat production. Stewed pork, a representative Chinese style meat product, is appreciated by consumers in most regions of China due to its simple processing technique [1] and distinct flavour [2]. Usually, the stewed pork is often produced by stewing the fresh pork in water with various condiments and spices for a long time [3]. Owing to the differences in the dietary habits of domestic consumers, the manufacturer would add seasonings to make different flavoured stewed meat products to meet the clients' needs. Some studies found that the seasonings created an enticing aroma during stewing and removed the undesirable odour in raw meat. Qin et al. [4] reported that a total of 37 volatile compounds were identified in stewed meat broths and the main volatile compounds, such as anethole, eucalptol, linalool, terpinen-4-ol, alpha-terpineol, and cedrol, may originate from star anise. More hexadecanal, octadecanal, and 9-octadecenal were present in soy sauce-stewed pork than in those water-boiled pork, and only 3,5-dimethyl-trans-1,2,4-trithiolane was detected in soy sauce-stewed pork [5]. It can be concluded that the addition of different 
food seasoning may have an important effect on the flavour formation of meat products. Although it seems reasonable that the flavour of stewed meat products would be associated with the addition of different food seasonings, there is currently little quantitative information available concerning the relationship between seasoning addition and the flavour of stewed meat.

To the best of our knowledge, salt, spices, soy sauce, sugar, and cooking wine are commonly used when cooking meat. Salt is a widely used additive in the meat industry due to its preservation and antimicrobial properties [6], and can accelerate lipid oxidation [7] to generate some lipid-related volatile compounds, such as linear-chain aldehydes and furans [8] during the heating treatment. The soy sauce and cooking wine contain many amino acids which may be the critical contributors to the characteristic flavour of the stewed pork [5,9]. Sugar, the flavour precursors to the Maillard reaction, usually plays an important role in the generation of aroma compounds of processed food. The studies had reported that the aldehydes, pyrazines and furans generated from Maillard reaction were considered as the key compounds of pot-stewed chicken meat products [10]. The formation of methyl aldehyde and pyrazine might come from the degradation of amino acids, which were partly associated with the reaction between reducing sugars and amino acids [11]. The spices are used as flavouring ingredient and natural antioxidants [12] in the processed meat products. For example, the addition of $0.5 \%$ garlic or onion before irradiation was effective in reducing lipid oxidation and provided some garlic/onion aromas for the cooked ground beef [13]. The addition of star anise changed the composition and proportion of volatile compounds, and imparted a spicy flavour to stewed chicken [14]. Although the important contribution of volatile compounds formed in common seasonings for meat products have been fully investigated, the sources and changes of flavour compounds produced by adding seasonings in the pork stewing process remain unknown.

A combination of gas chromatography-mass spectrometry (GC-MS) and olfactometric detector is used to connect the volatile compounds with sensory sniffing to more favourably and effectively identify the key odour-active compounds from numerous volatile constituents in the meat products, such as stewed pork broth [15] and braised pork [16]. Compared with gas chromatography-mass spectrometry/olfactometry (GC$\mathrm{MS} / \mathrm{O}$ ), comprehensive two-dimensional gas chromatography-time-flight mass spectrometry (GC $\times$ GC-TOFMS) not only offered higher separation power, but also maintained more desirable sensitivity [17]. The GC $\times$ GC-TOFMS has been considered as a powerful technique for detailed profiling of the flavour profile of different food stuffs, such as braised chicken [10], dry-cured ham [18], and green teas [19]. Additionally, the key characteristic compounds and volatile profiles of the fresh and grilled eel were investigated by electronic nose (E-nose), GC-O, GC-MS, and GC $\times$ GC-TOFMS [17]. In summary, the combination of GC-MS/O and GC $\times$ GC-TOFMS is meaningful and facilitative for the identification of major flavour compounds and analysis of flavour fingerprints.

The purpose of this study was to characterize the volatile profiles of stewed pork via GC-MS/O combined with GC $\times$ GC-TOFMS, and evaluate the effect of the addition of water, salt, spices, soy sauce, sugar and cooking wine on the volatile compounds and sensory properties of stewed pork. Moreover, the differences and changes of flavour compounds in stewed pork with the increase of seasonings would be fully understood, and the relationship between sensory evaluation and odour-active compounds in different stewed pork samples may be also systematically explored. Thus, different seasoning formulae could be used as a valuable direction to produce the stewed pork with a desirable flavour for the consumers.

\section{Materials and Methods}

\subsection{Sampling and Chemicals}

A total of 42 pieces of hind leg muscles with an average weight of approximately 0.8-1.0 kg from Duroc $\times$ (Landrace $\times$ Yorkshire) pig breed (aged 5-6 months and with body weights of 90-95 kg) were obtained from Chuying Agro-Pastoral Group Co. Ltd. 
(Zhengzhou, Henan Province, China). All pigs were reared under the same conditions, provided with the same feed and slaughtered following routine abattoir procedures (stunned, exsanguinated, scalded, dehaired and eviscerate). The hind leg muscles from the carcasses were cut into strips $(6.0 \mathrm{~cm} \times 4.0 \mathrm{~cm} \times 10.0 \mathrm{~cm})$ after removing visible fat and connective tissues. Then these muscles were packed in low-density polyethylene bags and stored at $-20{ }^{\circ} \mathrm{C}$. The sampling procedures were approved by the Animal Care and Use Committee of the Institute of Food Science and Technology, Chinese Academy of Agricultural Sciences, and performed in accordance with animal welfare and ethics. The 2-methyl-3-heptanone $(99 \%)$ and $n$-alkanes $\left(C_{7}-C_{30}\right)$ of chromatographic grade were bought from Sigma-Aldrich (Shanghai, China).

\subsection{Preparation of Stewed Pork Samples}

\subsubsection{Preparation of Spice Bag}

Allium fistulosum L. (45 g/ $\mathrm{kg}$ ) and Zingiber officinale Rosc. (30 g/ kg) were chopped evenly into pieces with the knife. $9.0 \mathrm{~g} / \mathrm{kg}$ Piper nigrum L., $1.2 \mathrm{~g} / \mathrm{kg}$ Cinnamomum cassia Presl., $0.3 \mathrm{~g} / \mathrm{kg}$ Syringa oblata Lindl., $1.0 \mathrm{~g} / \mathrm{kg}$ Myristica fragrans Houtt., $0.5 \mathrm{~g} / \mathrm{kg}$ Glycyrrhiza uralensis Fisch., $1.0 \mathrm{~g} / \mathrm{kg}$ llicium verum Hook.f., $0.3 \mathrm{~g} / \mathrm{kg}$ Cinnamomum wilsonii Gamble., $0.2 \mathrm{~g} / \mathrm{kg}$ Elettaria cardamomum (L.) Maton, $1.0 \mathrm{~g} / \mathrm{kg}$ Foeniculum vulgare Mill., $0.2 \mathrm{~g} / \mathrm{kg}$ Amomum kravanh Pierre ex Gagnep., $1.2 \mathrm{~g} / \mathrm{kg}$ Citrus reticulata Blanco, $0.8 \mathrm{~g} / \mathrm{kg}$ Alpinia officinarum Hance, $0.4 \mathrm{~g} / \mathrm{kg}$ Trifolium repens L., $0.6 \mathrm{~g} / \mathrm{kg}$ Piper longum Linn, $0.3 \mathrm{~g} / \mathrm{kg}$ Crataegus pinnatifida Bunge, $0.4 \mathrm{~g} / \mathrm{kg}$ Zanthoxylum bungeanum Maxim., $0.5 \mathrm{~g} / \mathrm{kg}$ Keampfera galangal L., $0.5 \mathrm{~g} / \mathrm{kg}$ Amomum tsaoko Crevost et Lemarie, and $1.2 \mathrm{~g} / \mathrm{kg}$ Angelica sinensis (per kg of pork) were ground into powder by the high-speed grinder and then mixed. All the above materials were put into one spice bag for stewed pork.

\subsubsection{Seasoning Formulations and Stewed Pork}

The pork strips were boiled in water for $10 \mathrm{~min}$ at $100^{\circ} \mathrm{C}$ to remove blood, and then stewed for $45 \mathrm{~min}$ at $98 \pm 2{ }^{\circ} \mathrm{C}$ with different seasoning formulations, finally soaked for $60 \mathrm{~min}$. The flow diagram of the stewed pork is shown in Figure S1. For stewed pork, the ratio of pork strip to seasoning formulation was 1:2 (w/v). In this study, there were six seasoning formulations to which the following treatments were randomly assigned (formulation 1:2 $\mathrm{L} / \mathrm{kg}$ water, formulation $2: 2 \mathrm{~L} / \mathrm{kg}$ water $+60 \mathrm{~g} / \mathrm{kg}$ salt, formulation $3: 2 \mathrm{~L} / \mathrm{kg}$ water $+60 \mathrm{~g} / \mathrm{kg}$ salt + one spice bag, formulation $4: 2 \mathrm{~L} / \mathrm{kg}$ water $+60 \mathrm{~g} / \mathrm{kg}$ salt + one spice bag $+20 \mathrm{~g} / \mathrm{kg}$ soy sauce, formulation $5: 2 \mathrm{~L} / \mathrm{kg}$ water $+60 \mathrm{~g} / \mathrm{kg}$ salt + one spice bag $+20 \mathrm{~g} / \mathrm{kg}$ soy sauce $+30 \mathrm{~g} / \mathrm{kg}$ sugar, formulation $6: 2 \mathrm{~L} / \mathrm{kg}$ water $+60 \mathrm{~g} / \mathrm{kg}$ salt + one spice bag $+20 \mathrm{~g} / \mathrm{kg}$ soy sauce $+30 \mathrm{~g} / \mathrm{kg}$ sugar $+30 \mathrm{~g} / \mathrm{kg}$ cooking wine). The water and seasoning were weighed based on each kilogram of pork. The stewed pork with formulations 1, 2, 3, 4, 5, and 6 were marked stewed pork one $\left(\mathrm{SP}_{1}\right)$, stewed pork two $\left(\mathrm{SP}_{2}\right)$, stewed pork three $\left(\mathrm{SP}_{3}\right)$, stewed pork four $\left(\mathrm{SP}_{4}\right)$, stewed pork five $\left(\mathrm{SP}_{5}\right)$ and stewed pork six $\left(\mathrm{SP}_{6}\right)$, respectively. It is worth noting that $\mathrm{SP}_{3}$ was cooked with seasoning formulation that referred to aged brine according to the following procedure [20]. The first brine (fresh brine) was obtained by removing the spice bag and stewed pork, subsequently, supplementing water, salt and spice bag into fresh brine, the second brine was obtained by the same procedures. Aged brine was eventually produced based on this cyclic process until the eighth cycle. The processing methods of seasoning formulations of $\mathrm{SP}_{4}, \mathrm{SP}_{5}$, and $\mathrm{SP}_{6}$ were the same as that of $\mathrm{SP}_{3}$. Only difference was the composition of seasoning formulation, which contained more soy sauce, sugar and cooking wine in sequence than $\mathrm{SP}_{3} . \mathrm{SP}_{1}$ and $\mathrm{SP}_{2}$ were cooked with water and salt-water. Fresh pork (FP) without stewing was used as the control group. All pork samples were collected the vacuum bags and stored at $-20^{\circ} \mathrm{C}$ until used.

\subsection{Extraction of Volatile Compounds}

The extraction of volatiles from the stewed pork was carried out using the manual solid-phase micro-extraction (SPME) equipped with a 50/30 $\mu$ m divinylbenzene/carboxen/ polydimethylsiloxane (DVB/CAR/PDMS) fibre (Supelco, Inc., Bellefonte, PA, USA). Briefly, 
$5.0 \mathrm{~g}$ of the pork sample was weighed precisely and placed in a $40 \mathrm{~mL}$ headspace vial. Immediately after, $1 \mu \mathrm{L}$ of 2-methyl-3-heptanone was added and sealed tightly with screw caps fitted with a Teflon/silicon septum. The vial was incubated in a thermostatic water bath at $60^{\circ} \mathrm{C}$ for $20 \mathrm{~min}$. The selected fibre was used to extract the volatile compounds in head space for $40 \mathrm{~min}$ at $60^{\circ} \mathrm{C}$. Upon completion, the fibre was inserted into the injection port $\left(250{ }^{\circ} \mathrm{C}\right)$ of the GC instrument to desorb the analyses for $5 \mathrm{~min}$.

\subsection{GC-MS/O Analysis}

The method was performed by the method of Han et al. [21] with minor modifications. The volatile compounds of stewed pork were analysed and identified by a GC-MS instrument (7890A-7000B, Agilent Technologise, Inc., Santa Clara, CA, USA) equipped with an olfactory detection port (Sniffer 9000; Brechbuhler, Schlieren, Switzerland). Capillary column DB-wax (30 m $\times 0.32 \mathrm{~mm}$ i.d., $0.25 \mu \mathrm{m}$ film thickness; J \& W Scientific, Inc., Folsom, CA, USA) was used with helium (purity of $\geq 99.999 \%$ ) as the carrier gas at $1.2 \mathrm{~mL} / \mathrm{min}$ flow rate. The front inlet temperature was $250{ }^{\circ} \mathrm{C}$ with a solvent delay of $4 \mathrm{~min}$. The temperature program was as follows: Oven temperature was maintained at $40^{\circ} \mathrm{C}$ for $3 \mathrm{~min}$, ramped to $200^{\circ} \mathrm{C}$ at a rate of 5 ${ }^{\circ} \mathrm{C} / \mathrm{min}$, then ramped to $240^{\circ} \mathrm{C}$ at a rate of $10^{\circ} \mathrm{C} / \mathrm{min}$ with a $3 \mathrm{~min}$ final hold. The infector mode was splitless. The transfer line temperature and ion source temperature were kept 240 ${ }^{\circ} \mathrm{C}$ and $230^{\circ} \mathrm{C}$. Electro-impact mass spectra were generated at $70 \mathrm{eV}$, with $\mathrm{m} / \mathrm{z}$ scan range from 50 to $400 \mathrm{amu}$. A sniffing port (Sniffer 9000) coupled to a GC-MS instrument was used for odour-active compound characterization. The effluent from the capillary column was split 1:1 $(v / v)$ between the mass spectrometry detector and the olfactory detector port. The eight trained staff were utilized for the sniffing test on GC-O. The volatile compounds were initially identified by the National Institute of Standards and Technology (NIST) Mass Spectral Library (Version 2.0). Subsequently, the identified compounds were further confirmed based on a comparison of GC retention indices (RI) with authentic compounds. Qualitative analysis was also performed according to odour properties.

\subsection{GC $\times$ GC-TOFMS Analysis}

The GC $\times$ GC-TOFMS system consists of an Agilent 7890 gas chromatography (Agilent Technologies, Palo Alto, CA, USA) equipped with cold-jet modulator and time-of-fight mass spectrometer (TOFMS; LECO Pegasus 4D). The first column was DB-WAX (30 m $\times 0.25 \mathrm{~mm}$ i.d. $\times 0.25 \mu \mathrm{m}$ film thickness) and the second column was DB-17HT ( $2 \mathrm{~m} \times$ $0.1 \mathrm{~mm}$ i.d. $\times 0.1 \mu \mathrm{m}$ film thickness). GC $\times$ GC conditions: the temperature of the injection port was $240{ }^{\circ} \mathrm{C}$; helium (99.999\%) flow, $1.0 \mathrm{~mL} / \mathrm{min}$; splitless injection; $6.0 \mathrm{~s}$ of modulation period; the column temperature program for the 1 st $\mathrm{D}$ column: initial temperature was $40{ }^{\circ} \mathrm{C}$ and held for $1 \mathrm{~min}$, increased to $220^{\circ} \mathrm{C}$ at $3.0^{\circ} \mathrm{C} / \mathrm{min}$, to $230^{\circ} \mathrm{C}$ at $10^{\circ} \mathrm{C} / \mathrm{min}$ and held for $5 \mathrm{~min}$; the column temperature program of the 2 nd D column: initial temperature at $45^{\circ} \mathrm{C}$ (held for $1 \mathrm{~min}$ ), increased to $225^{\circ} \mathrm{C}$ at $3.0^{\circ} \mathrm{C} / \mathrm{min}$ (held for $2.5 \mathrm{~min}$ ), to $230{ }^{\circ} \mathrm{C}$ at $10^{\circ} \mathrm{C} / \mathrm{min}$ (held for $5 \mathrm{~min}$ ). TOF-MS conditions: The ion source and transfer line to the mass spectrometer were maintained at $220^{\circ} \mathrm{C}$ and $290^{\circ} \mathrm{C}$, respectively. The ionization potential of MS was $70 \mathrm{eV}$, the detector voltage was $1620 \mathrm{~V}$, the scan range was 33 to $450 \mathrm{~m} / \mathrm{z}$, and the mass spectra data acquisition rate was 50 spectra/s. The identification of volatile compounds was based on the NIST 2014 library (Hewlett-Packard Co., Avondale, PA, USA), the mass spectral match factor $\geq 800$ and similarity $\geq 1000$.

\subsection{Quantification of Volatile Compounds}

The volatile compounds of stewed pork were semi-quantitated by the method of calibration with an internal standard (IS). The concentration of the volatile constituent was measured by the calibration curves of the GC-peak area and the amount ratios for the target analyst relative to 2-methyl-3-heptanone. The quantitative data of the identified compounds were obtained without considering the calibration factors, that is, the calibration 
factors were considered to be 1.00. The concentration of each compound was calculated as follows:

$$
\operatorname{Conc}\left(\frac{\mu \mathrm{g}}{\mathrm{kg}}\right)=\frac{\text { Peak area ratio }\left(\frac{\text { volatile }}{\mathrm{IS}}\right) \times 0.41 \mu \mathrm{g}(\mathrm{IS})}{5.0 \mathrm{~g}(\text { stewed pork })} \times 1000
$$

The odour activity value (OAV) of a compound was calculated as the ratio of its concentration in the stewed pork to its odour threshold in water. The equation is shown below:

$$
\mathrm{OAV}_{\mathrm{i}}=\frac{\mathrm{C}_{\mathrm{i}}}{\mathrm{OT}_{\mathrm{i}}}
$$

where $C_{i}$ is known as the concentration of the compound in the stewed pork and $\mathrm{OT}_{\mathrm{i}}$ is the odour threshold in water. Compounds with $\mathrm{OAV} \geq 1$ are considered to be the main contributors to the total flavour.

\subsection{Sensory Evaluation}

Sensory evaluation was carried out by 8 trained panellists ( 4 females and 4 males, aged 25-35 years). All assessors were recruited from Chinese Academy of Agriculture Sciences, Beijing and had at least one year of experience in the sensory descriptive analysis of stewed meat products. In order to be more familiar with the flavour characteristics of stewed pork, the 12 weeks of training sessions ( 2 times per week and $2 \mathrm{~h}$ per session) were conducted by assessors. The panellist have descripted and defined the flavour attributes, reference standard and intensities (Supplementary Table S1). Samples were coded with three-digit randomized numbers and presented to the assessors at room temperature. Panellists selected five flavour attributes to be evaluated, namely, fatty odour, meaty odour, caramel odour, soy sauce odour and spicy odour. Each attribute was scored on a $10 \mathrm{~cm}$ non-structured line with anchor points at each end $(0=$ not perceivable, $10=$ strongly perceivable) [22]. The mean value of sensory attributes was shown in the radar chart.

\subsection{Statistical Analysis}

The contents of volatile compounds and odour activity values (OAVs) of the odouractive compounds in stewed pork were presented as the mean \pm standard deviation (SD). Significant differences were determined by one-way analysis of variance (ANOVA) and Duncan's multiple range test at $p<0.05$ of statistical product and service solutions (SPSS) software (v. 19.0, SPSS, Inc., Chicago, IL, USA). The PCA and PLS-DA were performed based on the mean OAVs using the software XLSTAT (2016) from Addinsoft (Barcelona, Spain). The data of odour-active compounds and sensory description in different stewed pork was also conducted with PLSR of the software XLSTAT (2016) from Addinsoft (Barcelona, Spain). The heat maps of the correlation data of PLS-DA and PLSR were conducted by R v3.2.2 (R Studio Team, 2012).

\section{Results and Discussion}

\subsection{Analysis of Different Types of Volatile Components in Fresh and Stewed Pork}

The kinds and content ratios of volatile components in all pork samples were shown in Table 1, the most abundant volatile compounds were aldehydes, followed by hydrocarbons, alcohols, heterocyclic and sulphur compounds, finally ketones, ethers, phenols, esters, and acids. These kinds of volatile compounds have been also found in cooked pork products [16,21]. In terms of the stewed pork with different seasoning formulations, the types and proportions of aldehydes, heterocyclic and sulphur-containing compounds increased significantly when the pork was stewed in water and salt $\left(\mathrm{SP}_{1}\right.$ and $\left.\mathrm{SP}_{2}\right)$, while these compounds of pork samples treated with spices, soy sauce, sugar and cooking wine $\left(\mathrm{SP}_{3}, \mathrm{SP}_{4}, \mathrm{SP}_{5}\right.$, and $\left.\mathrm{SP}_{6}\right)$ decreased slightly. This result indicated that a large amount of flavour compounds was formed during the thermal processing of pork, however some of them were inhibited due to the addition of edible condiments. Additionally, it has been reported that the peak area and percentage 
composition of aldehydes increased in stewed chicken with the addition of star anise, whereas that of Maillard reaction products decreased [14]. The numbers of alcohols and ketones had gradually increased with the addition of seasoning in pork samples, and the content ratios of hydrocarbons in samples $\mathrm{SP}_{3}, \mathrm{SP}_{4}, \mathrm{SP}_{5}$, and $\mathrm{SP}_{6}$ were much higher than those of samples from other treatment groups. These analyse concluded that the heat treatment and seasoning play the important role to the flavour of the cooked pork [23,24]. Furthermore, compared with compounds detected by GC-MS/O, more volatile compounds (e.g., aldehydes, alcohols, ketones, ethers, acids, heterocyclic, and sulphur-containing compounds) were identified by GC $\times$ GC-TOFMS, while some long-chain aldehydes and hydrocarbons were only detected using GC-MS/O (Supplementary Table S2). The above results have shown that the GC $\times$ GC-TOFMS combined with GC-MS/O could more comprehensively analyse the volatile profile in the fresh and stewed pork.

Table 1. The comparison of kinds and content ratios of volatile compounds by gas chromatography-mass spectrometry/olfactometry (GC-MS/O) and two-dimensional gas chromatographic combined with time-of-fight mass spectrometry $(\mathrm{GC} \times \mathrm{GC}-\mathrm{TOFMS})$.

\begin{tabular}{|c|c|c|c|c|c|c|c|c|c|c|c|c|c|c|c|}
\hline & \multirow{2}{*}{ Group } & \multicolumn{7}{|c|}{ GC-MS/O } & \multicolumn{7}{|c|}{ GC $\times$ GC-TOFMS } \\
\hline & & FP & $\mathrm{SP}_{1}$ & $\mathrm{SP}_{2}$ & $\mathrm{SP}_{3}$ & $\mathrm{SP}_{4}$ & $\mathrm{SP}_{5}$ & $\mathrm{SP}_{6}$ & FP & $\mathrm{SP}_{1}$ & $\mathrm{SP}_{2}$ & $\mathrm{SP}_{3}$ & $\mathrm{SP}_{4}$ & $\mathrm{SP}_{5}$ & $\mathrm{SP}_{6}$ \\
\hline \multirow{10}{*}{ Quantities } & Aldehydes & 5 & 12 & 12 & 11 & 11 & 11 & 11 & 20 & 22 & 22 & 20 & 19 & 21 & 22 \\
\hline & Alcohols & 3 & 5 & 5 & 6 & 7 & 7 & 8 & 14 & 13 & 16 & 17 & 19 & 16 & 18 \\
\hline & Ketones & 0 & 0 & 0 & 3 & 3 & 3 & 3 & 8 & 10 & 12 & 13 & 11 & 14 & 13 \\
\hline & Hydrocarbons & 6 & 11 & 13 & 11 & 13 & 13 & 14 & 6 & 11 & 12 & 18 & 13 & 15 & 13 \\
\hline & Esters & 0 & 0 & 0 & 0 & 0 & 0 & 0 & 1 & 8 & 10 & 11 & 10 & 11 & 11 \\
\hline & Ethers & 1 & 2 & 1 & 3 & 4 & 4 & 4 & 1 & 1 & 2 & 5 & 5 & 5 & 5 \\
\hline & Phenols & 0 & 0 & 0 & 2 & 2 & 2 & 2 & 1 & 0 & 0 & 2 & 2 & 2 & 2 \\
\hline & Acids & 0 & 0 & 0 & 0 & 0 & 0 & 0 & 3 & 0 & 2 & 2 & 2 & 2 & 2 \\
\hline & $\begin{array}{l}\text { Heterocyclic and } \\
\text { sulfur compounds }\end{array}$ & 0 & 2 & 2 & 1 & 1 & 1 & 1 & 3 & 7 & 10 & 12 & 13 & 13 & 13 \\
\hline & Total & 15 & 32 & 33 & 37 & 41 & 41 & 43 & 57 & 72 & 86 & 100 & 94 & 99 & 99 \\
\hline \multirow{10}{*}{ Ratios (\%) } & Aldehydes & 37.8 & 66.8 & 63.7 & 34.9 & 40.2 & 37.8 & 43.2 & 43.1 & 30.6 & 38.9 & 20.0 & 17.9 & 18.5 & 19.9 \\
\hline & Alcohols & 9.1 & 11.8 & 12.1 & 26.3 & 21.1 & 21.4 & 18.5 & 13.5 & 13.3 & 6.2 & 15.8 & 7.8 & 8.1 & 6.6 \\
\hline & Ketones & 0.0 & 0.0 & 0.0 & 6.0 & 3.6 & 4.6 & 3.2 & 23.5 & 9.0 & 16.5 & 11.1 & 11.4 & 7.3 & 11.8 \\
\hline & Hydrocarbons & 48.4 & 12.3 & 16.7 & 18.9 & 24.6 & 23.7 & 25.4 & 16.8 & 29.1 & 19.7 & 40.7 & 50.1 & 47.3 & 48.9 \\
\hline & Esters & 0.0 & 0.0 & 0.0 & 0.0 & 0.0 & 0.0 & 0.0 & 0.1 & 2.8 & 5.2 & 2.5 & 3.7 & 9.1 & 4.4 \\
\hline & Ethers & 4.8 & 1.2 & 1.0 & 6.2 & 3.7 & 4.6 & 3.6 & 0.2 & 0.2 & 0.2 & 2.4 & 0.8 & 0.9 & 0.5 \\
\hline & Phenols & 0.0 & 0.0 & 0.0 & 2.9 & 3.8 & 4.5 & 2.7 & 0.01 & 0.0 & 0.0 & 0.5 & 0.5 & 0.7 & 0.2 \\
\hline & Acids & 0.0 & 0.0 & 0.0 & 0.0 & 0.0 & 0.0 & 0.0 & 2.7 & 0.3 & 0.5 & 0.4 & 0.3 & 0.6 & 0.5 \\
\hline & $\begin{array}{l}\text { Heterocyclic and } \\
\text { sulfur compounds }\end{array}$ & 0.0 & 7.9 & 6.4 & 4.9 & 3.0 & 3.3 & 3.4 & 0.1 & 14.7 & 12.9 & 6.5 & 7.5 & 7.5 & 7.0 \\
\hline & Total & 100.0 & 100.0 & 100.0 & 100.0 & 100.0 & 100.0 & 100.0 & 100.0 & 100.0 & 100.0 & 100.0 & 100.0 & 100.0 & 100.0 \\
\hline
\end{tabular}

Note: FP, fresh pork; SP1, stewed pork with water; SP2: stewed pork with water and salt; SP3: stewed pork with water, salt and spices; SP4: stewed pork with water, salt, spices and soy sauce; SP5: stewed pork with water, salt, spices, soy sauce and sugar; SP6: stewed pork with water, salt, spices, soy sauce, sugar and cooking wine.

\subsection{Volatile Compounds Profiling in the Fresh and Stewed Pork}

A total of 139 volatile compounds of the fresh and stewed pork were extracted and identified by GC-MS/O and GC × GC-TOFMS, including 30 aldehydes, 23 alcohols, 19 ketones, 25 hydrocarbons, 13 esters, five ethers, three phenols, four acids, and 17 heterocyclic and sulphur-containing compounds (Table 2). Most of these compounds have been reported in several pork products [24,25]. Aldehydes are mainly derived from the lipid oxidation and degradation reaction, and Strecker degradation of amino acids [21,26], which are considered as the major contributions to the overall flavour of meat products [20,27] due to their lower odour thresholds. Among seven stewed pork samples, the content of aldehydes was the most abundant, with the total mean amounts of $719.6-3326.1 \mu \mathrm{g} \cdot \mathrm{kg}^{-1}$. Obviously, the content of aldehydes in heated pork samples $\left(\mathrm{SP}_{1}, \mathrm{SP}_{2}, \mathrm{SP}_{3}, \mathrm{SP}_{4}, \mathrm{SP}_{5}\right.$, and $\left.\mathrm{SP}_{6}\right)$ were significantly greater $(p<0.001)$ than those in the fresh pork. This indicated that the thermal 
processing of pork products plays a crucial role in the formation of aldehydes [23]. It can be also found that 15 compounds, namely, nine saturated aldehydes (butanal, pentanal, hexanal, heptanal, octanal, nonanal, decanal, pentadecanal and (E)-octadec-9-enal), four olefin aldehydes ( $(E)$-oct-2-enal, $(E)$-non-2-enal, $(Z)$-dec-4-enal and $(E)$-dec-2-enal), as well as two aromatic aldehydes (benzaldehyde and benzeneacetaldehyde) were simultaneously identified in all stewed pork samples. The saturated and olefin aldehydes could be produced from the degradation of fatty acids [28] and the aromatic aldehydes were usually generated from Strecker reaction [29]. Among them, except for pentanal and benzeneacetaldehyde, the contents of other 13 compounds were significantly higher $(p<0.001)$ in $\mathrm{SP}_{1}$ and $\mathrm{SP}_{2}$ than those in $\mathrm{SP}_{3}, \mathrm{SP}_{4}, \mathrm{SP}_{5}$, and $\mathrm{SP}_{6}$. Moreover, (Z)-hept-2-enal, 2-butyloct-2-enal, and tertradecanal were detected only in $\mathrm{SP}_{1}$ and $\mathrm{SP}_{2}$. It was shown that the spices, soy sauce, sugar, and cooking wine may have an inhibitory effect on the production of some aldehydes in stewed pork. Moreover, 4-(1-methylethyl)-benzaldehyde, p-anisaldehyde and cinnamaldehyde, with mint and sweet aroma [20], could be generated from the Chinese different spices, including fennel, anise, and cinnamon, respectively.

Alcohols, with pleasant fruity and floral notes [18], are mainly derived from spices [30] and the oxidative decomposition of lipid [31]. For the identified alcohols, 1,8-cineole, pentan-1-ol, hexan-1-ol, oct-1-en-3-ol, heptan-1-ol, 2-etylhexan-1-ol, octan-1-ol and (E)oct-2-en-1-ol could be detected in all pork samples. Among them, the linear alcohols (pentan-1-ol, hexan-1-ol, heptan-1-ol, octan-1-ol and (E)-oct-2-en-1-ol) and branched alcohols (oct-1-en-3-ol and 2-etylhexan-1-ol) were the main lipid oxidation products. Meanwhile, the contents of these compounds were significantly higher $(p<0.01)$ in $\mathrm{SP}_{1}$ and $\mathrm{SP}_{2}$ than those in $\mathrm{FP}, \mathrm{SP}_{3}, \mathrm{SP}_{4}, \mathrm{SP}_{5}$ and $\mathrm{SP}_{6}$. The 1,8-cineole, linalool, terpinen-4-ol, terpineol, $(4 \mathrm{R}, 6 \mathrm{R})-2-$ undecen-1-ol and cis-geraniol increased significantly $(p<0.001)$ in $\mathrm{SP}_{3}$ as the spices were added in the stewed pork and greatly reduced in $\mathrm{SP}_{4}, \mathrm{SP}_{5}$ and $\mathrm{SP}_{6}$. This result shows that these above compounds were mainly formed from aged brine (spices, soy sauce, sugar and cooking wine).

Ketones are often considered to have a great influence on the aroma of meat and meat products since they are presented in large amounts and exhibited specific aroma in food [32]. As shown in Table 2, 7 ketones such as 6-methylhept-5-en-2-one, 4-isopropyl-2cyclohexenone, piperitone, carvone, 4-phenylbutan-2-one, 4-methoxyphenylacetone and xanthoxylin were detected in the spice, soy sauce, sugar and cooking wine processed samples $\left(\mathrm{SP}_{3}, \mathrm{SP}_{4}, \mathrm{SP}_{5}\right.$ and $\left.\mathrm{SP}_{6}\right)$, and they were not detected in fresh pork (FP), stewed pork with water or salt $\left(\mathrm{SP}_{1}\right.$ or $\left.\mathrm{SP}_{2}\right)$. It indicated that more ketone compounds were formed due to the addition of food seasoning, which provided a richer fruity and nutty aroma [25] for the overall pork flavour. Pham et al. [33] reported that the methyl ketones were considered as the precursors to the fatty aromas related to cooked meat, which could be formed by the oxidative degradation of fatty acids [34]. In this study, the methyl ketones (e.g., heptan-2-one, octan-2-one, pentane-2,3-dione, octane-2,3-dione, 3-octen-2-one, $(E, E)-3,5$ octadien-2-one, 6-methyl-3,5-heptadiene-2-one and acetophenone) were originated from the oxidation of lipids during heating.

There were few hydrocarbons detected in the fresh pork, however more hydrocarbons could be formed by added water, salt, soy sauce, sugar and cooking wine during the pork processing. All hydrocarbons could be divided into aromatic hydrocarbons and aliphatic hydrocarbons. Among them, 16 aromatic hydrocarbons had been identified in all stewed pork samples, and toluene, ethylbenzene, 1,3-dimethylbenzene, o-xylene and styrene were presented as common compounds. The production of toluene and ethylbenzene primarily come from amino acid degradation. This result was consistent with that reported by Olivares et al. [11]. It was also found that nine aliphatic hydrocarbons were detected in stewed pork samples $\left(\mathrm{SP}_{1}, \mathrm{SP}_{2}, \mathrm{SP}_{3}, \mathrm{SP}_{4}, \mathrm{SP}_{5}\right.$ and $\left.\mathrm{SP}_{6}\right)$. Previous study has shown that aliphatic hydrocarbons had a limited influence on aroma perception due to their high threshold values [35] and raised mainly from lipid oxidation [27]. Additionally, phellandrene, (Z)-3,7dimethyl-1,3,6-octatriene, o-cymene, m-cymene, 1-methylindan, and longifolene could be formed from the added spices and soy sauce during the processing. 
Table 2. Concentrations and origin of volatile compounds of the fresh and stewed pork.

\begin{tabular}{|c|c|c|c|c|c|c|c|c|c|c|}
\hline No. & Compounds & FP & $\mathrm{SP}_{1}$ & $\mathrm{SP}_{2}$ & $\mathrm{SP}_{3}$ & $\mathrm{SP}_{4}$ & $\mathrm{SP}_{5}$ & $\mathrm{SP}_{6}$ & $p$ Value & Origin $^{1}$ \\
\hline & Aldehydes (30) & $719.6 \pm 36.8^{d}$ & $2478.5 \pm 104.6^{b}$ & $3326.1 \pm 219.3^{a}$ & $959.1 \pm 1.9^{c}$ & $795.9 \pm 7.8^{\mathrm{cd}}$ & $815.1 \pm 25.8^{\mathrm{cd}}$ & $934.2 \pm 44.1^{c}$ & 0.000 & \\
\hline 1 & Acetaldehyde & $1.7 \pm 0.2^{a}$ & N.D. & N.D. & N.D. & N.D. & $1.7 \pm 0.3^{a}$ & $0.9 \pm 0.1^{b}$ & 0.011 & AADP \\
\hline 2 & Propanal & N.D. & N.D. & $97.9 \pm 15.0^{a}$ & N.D. & $16.1 \pm 3.2^{b}$ & $25.5 \pm 1.7^{b}$ & $29.3 \pm 2.4^{b}$ & 0.000 & AADP \\
\hline 3 & Butanal & $0.5 \pm 0.2^{d}$ & $3.7 \pm 0.8^{b}$ & $19.0 \pm 1.5^{\mathrm{a}}$ & $2.1 \pm 0.9^{c}$ & $3.5 \pm 0.6^{b}$ & $2.0 \pm 0.3^{c}$ & $2.0 \pm 0.3^{c}$ & 0.000 & LOP \\
\hline 4 & Pentanal & $27.5 \pm 4.0^{\mathrm{e}}$ & $53.6 \pm 6.9^{d}$ & $74.3 \pm 1.3^{\mathrm{ab}}$ & $80.7 \pm 3.8^{a}$ & $60.4 \pm 9.8^{\mathrm{cd}}$ & $70.0 \pm 5.6^{\mathrm{bc}}$ & $65.7 \pm 3.7^{b c}$ & 0.000 & LOP \\
\hline 5 & Hexanal & $3.5 \pm 1.0^{\mathrm{e}}$ & $633.2 \pm 13.3^{a}$ & $634.1 \pm 14.7^{\mathrm{a}}$ & $233.2 \pm 8.9^{c}$ & $253.8 \pm 10.1^{b}$ & $176.4 \pm 10.5^{\mathrm{d}}$ & $221.2 \pm 15.4^{c}$ & 0.000 & LOP \\
\hline 6 & Heptanal & $56.7 \pm 3.4^{c}$ & $122.6 \pm 15.7^{b}$ & $160.1 \pm 2.1^{a}$ & $31.5 \pm 1.7^{\mathrm{d}}$ & $36.9 \pm 1.2^{\mathrm{d}}$ & $38.3 \pm 3.6^{d}$ & $38.2 \pm 7.2^{d}$ & 0.000 & LOP \\
\hline 7 & Octanal & $84.7 \pm 10.9^{c}$ & $182.0 \pm 10.3^{b}$ & $230.9 \pm 25.1^{a}$ & $62.4 \pm 5.0^{\mathrm{d}}$ & $86.4 \pm 9.7^{c}$ & $50.5 \pm 4.3^{d}$ & $54.9 \pm 8.6^{d}$ & 0.000 & LOP \\
\hline 8 & (Z)-Hept-2-enal & N.D. & $5.6 \pm 0.2^{b}$ & $38.5 \pm 9.0^{\mathrm{a}}$ & N.D. & N.D. & N.D. & N.D. & 0.024 & LOP \\
\hline 9 & 2-Methylpentanal & N.D. & $3.6 \pm 0.1^{a}$ & N.D. & $1.7 \pm 0.3^{b}$ & N.D. & N.D. & $1.7 \pm 0.01^{b}$ & 0.000 & LOP \\
\hline 10 & Nonanal & $219.8 \pm 13.3^{\mathrm{cd}}$ & $1475.5 \pm 124.6^{a}$ & $844.4 \pm 94.8^{b}$ & $316.3 \pm 5.8^{c}$ & $171.4 \pm 17.8^{\mathrm{d}}$ & $110.3 \pm 7.9^{d}$ & $284.2 \pm 11.9^{c}$ & 0.000 & LOP \\
\hline 11 & (E)-Oct-2-enal & $24.6 \pm 1.1^{\mathrm{c}}$ & $76.0 \pm 1.2^{a}$ & $42.2 \pm 7.4^{b}$ & $3.2 \pm 0.2^{d}$ & $5.5 \pm 1.1^{\mathrm{d}}$ & $6.6 \pm 1.8^{d}$ & $8.3 \pm 0.6^{\mathrm{d}}$ & 0.000 & LOP \\
\hline 13 & Benzaldehyde & $194.4 \pm 17.0^{\mathrm{c}}$ & $315.1 \pm 13.5^{b}$ & $370.6 \pm 52.7^{a}$ & $130.4 \pm 12.0$ de & $110.0 \pm 8.5^{\mathrm{e}}$ & $200.4 \pm 23.3^{c}$ & $156.7 \pm 11.5^{\mathrm{cd}}$ & 0.000 & AADP \\
\hline 14 & (E)-Non-2-enal & $7.5 \pm 0.9^{c}$ & $11.3 \pm 2.5^{b}$ & $15.1 \pm 1.0^{\mathrm{a}}$ & $1.6 \pm 0.1^{d}$ & $1.8 \pm 0.7^{\mathrm{d}}$ & $2.4 \pm 0.4^{d}$ & $1.9 \pm 0.4^{\mathrm{d}}$ & 0.000 & LOP \\
\hline 15 & (Z)-Dec-4-enal & $11.9 \pm 1.0^{\mathrm{a}}$ & $14.1 \pm 2.8^{a}$ & $14.2 \pm 2.3^{a}$ & $3.4 \pm 0.3^{b}$ & $3.6 \pm 0.4^{b}$ & $4.0 \pm 0.4^{b}$ & $3.0 \pm 0.1^{b}$ & 0.000 & LOP \\
\hline 16 & Benzeneacetaldehyde & $2.3 \pm 0.1^{\mathrm{d}}$ & $4.2 \pm 0.9^{c}$ & $5.8 \pm 1.6^{\text {be }}$ & $3.6 \pm 0.2^{\mathrm{cd}}$ & $7.4 \pm 0.4^{b}$ & $9.5 \pm 1.3^{a}$ & $6.5 \pm 0.4^{b}$ & 0.000 & MRP \\
\hline 17 & $(E)$-Dec-2-enal & $2.9 \pm 0.2^{b}$ & $5.6 \pm 1.1^{\mathrm{a}}$ & $4.6 \pm 1.0^{\mathrm{a}}$ & $1.0 \pm 0.1^{\mathrm{c}}$ & $0.6 \pm 0.02^{c}$ & $0.7 \pm 0.1^{c}$ & $0.8 \pm 0.1^{\mathrm{c}}$ & 0.000 & LOP \\
\hline 18 & 2-Butyloct-2-enal & N.D. & $6.3 \pm 0.3^{b}$ & $10.1 \pm 1.4^{\mathrm{a}}$ & N.D. & N.D. & N.D. & N.D. & 0.038 & LOP \\
\hline 19 & $(E, E)-2,4$-Nonadienal & $2.7 \pm 0.3^{b}$ & $6.0 \pm 0.3^{a}$ & $5.8 \pm 0.2^{a}$ & N.D. & N.D. & N.D. & N.D. & 0.000 & LOP \\
\hline 20 & Undec-2-enal & $2.5 \pm 0.2^{a}$ & $2.7 \pm 0.7^{a}$ & $2.4 \pm 0.3^{a}$ & N.D. & N.D. & N.D. & N.D. & 0.717 & LOP \\
\hline 21 & $\begin{array}{l}\text { 4-(1-Methylethyl)- } \\
\text { benzaldehyde }\end{array}$ & N.D. & N.D. & N.D. & $1.2 \pm 0.1^{a}$ & $0.7 \pm 0.2^{c}$ & $0.9 \pm 0.1^{b}$ & $0.5 \pm 0.1^{c}$ & 0.001 & VFAB \\
\hline 22 & $(E, E)-2,4$-Decadienal & $7.3 \pm 1.0^{b}$ & $10.0 \pm 0.5^{\mathrm{a}}$ & $7.9 \pm 0.9^{b}$ & $0.8 \pm 0.01^{\mathrm{c}}$ & N.D. & $0.9 \pm 0.2^{c}$ & $1.6 \pm 0.1^{\mathrm{c}}$ & 0.000 & LOP \\
\hline
\end{tabular}


Table 2. Cont.

\begin{tabular}{|c|c|c|c|c|c|c|c|c|c|c|}
\hline No. & Compounds & FP & $\mathrm{SP}_{1}$ & $\mathrm{SP}_{2}$ & $\mathrm{SP}_{3}$ & $\mathrm{SP}_{4}$ & $\mathrm{SP}_{5}$ & $\mathrm{SP}_{6}$ & $p$ Value & Origin $^{1}$ \\
\hline 23 & p-Anisaldehyde & N.D. & N.D. & N.D. & $3.0 \pm 0.2^{b}$ & $1.4 \pm 0.2^{\mathrm{d}}$ & $3.5 \pm 0.2^{\mathrm{a}}$ & $2.4 \pm 0.4^{c}$ & 0.000 & VFAB \\
\hline 24 & Cinnamaldehyde & N.D. & N.D. & N.D. & $2.0 \pm 0.4^{\mathrm{ab}}$ & $1.1 \pm 0.2^{b}$ & $2.5 \pm 0.2^{a}$ & $2.3 \pm 0.8^{a}$ & 0.031 & VFAB \\
\hline 26 & Undec-10-enal & $0.6 \pm 0.1^{b}$ & $0.3 \pm 0.1^{\mathrm{c}}$ & $1.7 \pm 0.2^{\mathrm{a}}$ & N.D. & N.D. & N.D. & N.D. & 0.000 & LOP \\
\hline 27 & (E)-Octadec-9-enal & $0.5 \pm 0.01^{\mathrm{d}}$ & $4.9 \pm 0.5^{\mathrm{a}}$ & $1.7 \pm 0.1^{b}$ & $1.1 \pm 0.1^{\mathrm{c}}$ & $1.1 \pm 0.1^{\mathrm{c}}$ & $1.9 \pm 0.1^{b}$ & $1.2 \pm 0.4^{c}$ & 0.000 & LOP \\
\hline 28 & $\beta$-Cyclocitral & $1.1 \pm 0.02^{b}$ & N.D. & N.D. & N.D. & $4.2 \pm 1.2^{\mathrm{a}}$ & $3.5 \pm 0.2^{a}$ & $1.9 \pm 0.2^{b}$ & 0.001 & LOP \\
\hline 29 & Tetradecanal & N.D. & $5.2 \pm 0.8^{a}$ & $4.8 \pm 0.5^{\mathrm{a}}$ & $2.7 \pm 0.1^{b}$ & N.D. & N.D. & N.D. & 0.003 & LOP \\
\hline \multirow[t]{2}{*}{30} & Hexadecanal & N.D. & $31.1 \pm 0.8^{c}$ & $34.7 \pm 0.2^{b}$ & $40.4 \pm 0.8^{a}$ & $7.2 \pm 0.3^{f}$ & $19.6 \pm 1.1^{\mathrm{d}}$ & $14.4 \pm 0.6^{\mathrm{e}}$ & 0.000 & LOP \\
\hline & Alcohols (23) & $224.8 \pm 14.1^{\mathrm{f}}$ & $1073.6 \pm 22.0^{a}$ & $525.9 \pm 29.8^{c}$ & $756.6 \pm 10.2^{b}$ & $347.5 \pm 14.7^{\mathrm{d}}$ & $358.1 \pm 1.0^{\mathrm{d}}$ & $310.2 \pm 6.6^{\mathrm{e}}$ & 0.000 & \\
\hline 31 & 4-Methylpentan-1-ol & N.D. & N.D. & $3.0 \pm 0.2^{b}$ & N.D. & N.D. & $6.2 \pm 0.2^{a}$ & $2.3 \pm 0.1^{c}$ & 0.000 & $\mathrm{UO}$ \\
\hline 32 & Pent-1-en-3-ol & N.D. & $17.1 \pm 2.7^{a}$ & $16.2 \pm 2.7^{a}$ & N.D. & $2.9 \pm 0.4^{c}$ & N.D. & N.D. & 0.000 & LOP \\
\hline 33 & Butan-1-ol & $1.5 \pm 0.2^{c}$ & N.D. & $5.6 \pm 1.1^{a}$ & $1.7 \pm 0.1^{b c}$ & $1.8 \pm 0.2^{b c}$ & $2.5 \pm 0.5^{b c}$ & $2.5 \pm 0.3^{b}$ & 0.000 & LOP \\
\hline 35 & Pentan-1-ol & $32.2 \pm 4.6^{c}$ & $268.2 \pm 17.6^{\mathrm{a}}$ & $165.8 \pm 13.9^{b}$ & $26.6 \pm 5.4^{c}$ & $26.3 \pm 3.3^{c}$ & $29.2 \pm 3.7^{c}$ & $28.6 \pm 0.4^{\mathrm{c}}$ & 0.000 & LOP \\
\hline 36 & (Z)-Pent-2-en-1-ol & N.D. & $1.3 \pm 0.3^{\mathrm{a}}$ & $1.5 \pm 0.1^{\mathrm{a}}$ & N.D. & N.D. & N.D. & N.D. & 0.312 & LOP \\
\hline 37 & Hexan-1-ol & $85.4 \pm 3.5^{b}$ & $477.1 \pm 18.3^{a}$ & $41.3 \pm 2.5^{c}$ & $2.8 \pm 0.6^{\mathrm{e}}$ & $6.1 \pm 0.7^{\mathrm{e}}$ & $24.3 \pm 2.6^{\mathrm{d}}$ & $6.6 \pm 0.5^{\mathrm{e}}$ & 0.000 & LOP \\
\hline 38 & Oct-1-en-3-ol & $1.5 \pm 0.4^{g}$ & $130.0 \pm 4.3^{\mathrm{b}}$ & $137.0 \pm 3.1^{\mathrm{a}}$ & $50.6 \pm 0.5^{d}$ & $59.4 \pm 0.4^{c}$ & $27.7 \pm 2.0^{\mathrm{f}}$ & $40.2 \pm 3.7^{\mathrm{e}}$ & 0.000 & LOP \\
\hline 39 & Heptan-1-ol & $10.3 \pm 1.6^{c}$ & $31.6 \pm 4.9^{\mathrm{a}}$ & $24.9 \pm 3.0^{b}$ & $3.3 \pm 0.4^{\mathrm{d}}$ & $4.8 \pm 3.6^{\mathrm{d}}$ & $2.5 \pm 0.2^{\mathrm{d}}$ & $3.7 \pm 0.2^{d}$ & 0.000 & LOP \\
\hline 40 & 2-Ethylhexan-1-ol & $8.7 \pm 2.3^{b}$ & $8.1 \pm 1.3^{b}$ & $11.6 \pm 0.9^{a}$ & $5.1 \pm 0.2^{c}$ & $5.6 \pm 0.3^{c}$ & $7.9 \pm 0.3^{b}$ & $5.3 \pm 0.8^{c}$ & 0.000 & LOP \\
\hline 41 & Linalool & N.D. & N.D. & N.D. & $75.6 \pm 5.9^{a}$ & $23.0 \pm 2.0^{b}$ & $22.3 \pm 0.9^{b}$ & $21.8 \pm 2.7^{b}$ & 0.000 & VFAB \\
\hline 42 & Octan-1-ol & $24.6 \pm 0.4^{c}$ & $49.4 \pm 5.8^{a}$ & $33.9 \pm 3.3^{b}$ & $9.8 \pm 0.4^{\mathrm{d}}$ & $5.9 \pm 0.7^{\mathrm{d}}$ & $8.0 \pm 0.9^{d}$ & $7.5 \pm 0.2^{\mathrm{d}}$ & 0.000 & LOP \\
\hline 43 & Butane-2,3-diol & N.D. & N.D. & $4.0 \pm 0.7^{b}$ & N.D. & $6.8 \pm 0.7^{a}$ & N.D. & N.D. & 0.007 & LOP \\
\hline 44 & Terpinen-4-ol & N.D. & N.D. & N.D. & $313.3 \pm 5.9^{a}$ & $93.2 \pm 6.8^{b}$ & $87.9 \pm 4.0^{b}$ & $71.7 \pm 3.5^{c}$ & 0.000 & VFAB \\
\hline 45 & (E)-Oct-2-en-1-ol & $41.8 \pm 6.2^{c}$ & $73.7 \pm 11.9^{a}$ & $59.3 \pm 10.0^{b}$ & $13.8 \pm 1.4^{\mathrm{d}}$ & $13.8 \pm 1.7^{\mathrm{d}}$ & $14.4 \pm 2.0^{\mathrm{d}}$ & $12.8 \pm 0.4^{\mathrm{d}}$ & 0.001 & LOP \\
\hline 46 & Nonan-1-ol & $6.3 \pm 0.8^{a}$ & $1.7 \pm 0.2^{b}$ & $0.6 \pm 0.01^{c}$ & $0.3 \pm 0.1^{\mathrm{c}}$ & $0.2 \pm 0.01^{c}$ & N.D. & $0.2 \pm 0.1^{\mathrm{c}}$ & 0.000 & LOP \\
\hline
\end{tabular}


Table 2. Cont.

\begin{tabular}{|c|c|c|c|c|c|c|c|c|c|c|}
\hline No. & Compounds & FP & $\mathrm{SP}_{1}$ & $\mathrm{SP}_{2}$ & $\mathrm{SP}_{3}$ & $\mathrm{SP}_{4}$ & $\mathrm{SP}_{5}$ & $\mathrm{SP}_{6}$ & $p$ Value & Origin $^{1}$ \\
\hline 47 & Terpineol & N.D. & N.D. & N.D. & $113.2 \pm 3.5^{\mathrm{a}}$ & $21.0 \pm 2.1^{d}$ & $52.5 \pm 1.9^{b}$ & $45.4 \pm 1.4^{\mathrm{c}}$ & 0.000 & VFAB \\
\hline 48 & (E)-Undec-2-en-1-ol & $4.8 \pm 0.8^{\mathrm{ab}}$ & $5.5 \pm 0.6^{a}$ & $5.2 \pm 1.3^{\mathrm{ab}}$ & $2.9 \pm 0.2^{\mathrm{cd}}$ & $3.8 \pm 0.5^{b c}$ & N.D. & $2.2 \pm 1.1^{\mathrm{d}}$ & 0.002 & LOP \\
\hline 50 & cis-Geraniol & N.D. & N.D. & N.D. & $11.3 \pm 1.7^{\mathrm{a}}$ & $7.4 \pm 0.6^{b}$ & $3.4 \pm 0.1^{c}$ & $7.7 \pm 0.8^{b}$ & 0.000 & VFAB \\
\hline 51 & Phenylethyl alcohol & $2.7 \pm 0.2^{b}$ & N.D. & $0.8 \pm 0.02^{d}$ & $2.3 \pm 0.1^{b}$ & $1.4 \pm 0.3^{c}$ & $2.2 \pm 0.1^{b}$ & $8.3 \pm 0.5^{a}$ & 0.000 & UO \\
\hline 52 & Undecan-1-ol & $3.0 \pm 0.4$ & N.D. & N.D. & N.D. & N.D. & N.D. & N.D. & N.D. & VFRM \\
\hline \multirow[t]{2}{*}{53} & Dodecan-1-ol & $0.3 \pm 0.01^{a}$ & $0.3 \pm 0.01^{a}$ & N.D. & N.D. & N.D. & N.D. & N.D. & 0.467 & VFRM \\
\hline & Ketones (19) & $377.2 \pm 17.6^{\text {de }}$ & $731.7 \pm 42.2^{b}$ & $1411.9 \pm 198.7^{a}$ & $530.8 \pm 16.6^{\mathrm{cd}}$ & $506.6 \pm 7.2^{\mathrm{cd}}$ & $323.6 \pm 1.2^{\mathrm{e}}$ & $555.9 \pm 16.2^{\mathrm{c}}$ & 0.000 & \\
\hline 54 & Butan-2-one & $1.3 \pm 0.3^{\mathrm{d}}$ & $2.7 \pm 0.9^{d}$ & $4.0 \pm 0.7^{\mathrm{cd}}$ & $30.5 \pm 4.9^{b}$ & $35.0 \pm 7.0^{b}$ & $9.3 \pm 0.7^{c}$ & $49.0 \pm 2.9^{a}$ & 0.000 & VFAB \\
\hline 55 & Butane-2,3-dione & $12.5 \pm 2.0^{\mathrm{bc}}$ & $23.6 \pm 1.7^{a}$ & $23.3 \pm 3.0^{\mathrm{a}}$ & $24.7 \pm 2.1^{a}$ & N.D. & $15.0 \pm 1.3^{b}$ & $11.1 \pm 0.5^{\mathrm{c}}$ & 0.000 & MRP \\
\hline 56 & Pentane-2,3-dione & N.D. & $15.3 \pm 2.9^{\mathrm{a}}$ & $18.0 \pm 2.6^{\mathrm{a}}$ & N.D. & N.D. & $4.2 \pm 0.6^{c}$ & $10.1 \pm 0.4^{b}$ & 0.000 & LOP \\
\hline 57 & Heptan-2-one & $25.2 \pm 2.6^{d}$ & $60.3 \pm 8.5^{c}$ & $118.5 \pm 11.2^{\mathrm{a}}$ & $19.4 \pm 1.7^{\mathrm{d}}$ & N.D. & $57.3 \pm 4.1^{c}$ & $72.1 \pm 5.8^{b}$ & 0.000 & LOP \\
\hline 59 & Octan-2-one & $7.2 \pm 1.7^{c}$ & $12.0 \pm 3.1^{b}$ & $18.3 \pm 0.6^{\mathrm{a}}$ & N.D. & N.D. & N.D. & N.D. & 0.002 & LOP \\
\hline 60 & $\begin{array}{l}\text { 1-Hydroxypropan-2- } \\
\text { one }\end{array}$ & N.D. & $20.7 \pm 3.6^{\mathrm{d}}$ & $57.7 \pm 15.0^{b}$ & $29.7 \pm 1.7^{\mathrm{d}}$ & $54.7 \pm 6.7^{b c}$ & $73.2 \pm 2.2^{a}$ & $43.0 \pm 2.2^{\mathrm{c}}$ & 0.000 & MRP \\
\hline 61 & Octane-2,3-dione & $303.7 \pm 20.8^{c}$ & $578.6 \pm 26.4^{b}$ & $1122.0 \pm 184.4^{\mathrm{a}}$ & $321.4 \pm 9.1^{\mathrm{c}}$ & $339.3 \pm 7.4^{\mathrm{c}}$ & N.D. & $297.9 \pm 16.0^{c}$ & 0.000 & LOP \\
\hline 62 & $\begin{array}{l}\text { 6-Methylhept-5-en-2- } \\
\text { one }\end{array}$ & N.D. & N.D. & N.D. & $3.8 \pm 0.5^{c}$ & $5.2 \pm 1.0^{\mathrm{a}}$ & $4.0 \pm 0.6^{b c}$ & $2.9 \pm 0.1^{c}$ & 0.019 & VFAB \\
\hline 63 & (E)-Oct-3-en-2-one & $4.3 \pm 0.6^{c}$ & $6.0 \pm 0.5^{b}$ & $10.2 \pm 0.6^{\mathrm{a}}$ & N.D. & N.D. & N.D. & N.D. & 0.000 & LOP \\
\hline 64 & $\begin{array}{c}(E, E)-3,5-\text { Octadien-2- } \\
\text { one }\end{array}$ & N.D. & N.D. & $24.1 \pm 3.1$ & N.D. & N.D. & N.D. & N.D. & N.D. & LOP \\
\hline 65 & $\begin{array}{c}\text { 6-Methyl-3,5- } \\
\text { heptadiene-2-one }\end{array}$ & N.D. & N.D. & $0.9 \pm 0.2^{a}$ & N.D. & N.D. & $0.3 \pm 0.01^{b}$ & N.D. & 0.003 & LOP \\
\hline 66 & Acetophenone & $2.2 \pm 0.2^{b c}$ & $4.2 \pm 0.9^{a}$ & $2.9 \pm 0.3^{b}$ & $2.6 \pm 0.2^{b c}$ & $2.0 \pm 0.1^{\mathrm{c}}$ & $2.7 \pm 0.5^{b c}$ & $2.3 \pm 0.2^{b c}$ & 0.001 & LOP \\
\hline 67 & $\begin{array}{l}\text { 4-Isopropyl-2- } \\
\text { cyclohexenone }\end{array}$ & N.D. & N.D. & N.D. & $2.0 \pm 0.1^{\mathrm{a}}$ & $1.2 \pm 0.1^{b}$ & $2.1 \pm 0.3^{a}$ & $1.4 \pm 0.2^{b}$ & 0.002 & VFAB \\
\hline
\end{tabular}


Table 2. Cont.

\begin{tabular}{|c|c|c|c|c|c|c|c|c|c|c|}
\hline No. & Compounds & FP & $\mathrm{SP}_{1}$ & $\mathrm{SP}_{2}$ & $\mathrm{SP}_{3}$ & $\mathrm{SP}_{4}$ & $\mathrm{SP}_{5}$ & $\mathrm{SP}_{6}$ & $p$ Value & Origin $^{1}$ \\
\hline 68 & Piperitone & N.D. & N.D. & N.D. & $54.5 \pm 2.9^{a}$ & $38.6 \pm 4.2^{b}$ & $41.5 \pm 3.3^{b}$ & $37.5 \pm 1.8^{b}$ & 0.001 & VFAB \\
\hline 69 & Carvone & N.D. & N.D. & N.D. & $2.7 \pm 0.3^{a}$ & $1.6 \pm 0.2^{b}$ & N.D. & N.D. & 0.005 & VFAB \\
\hline 71 & $\begin{array}{c}4- \\
\text { Methoxyphenylacetone }\end{array}$ & N.D. & N.D. & N.D. & $0.7 \pm 0.02^{a}$ & $0.4 \pm 0.1^{b}$ & $0.8 \pm 0.1^{a}$ & $0.4 \pm 0.0^{\mathrm{b}}$ & 0.006 & VFAB \\
\hline \multirow[t]{2}{*}{72} & Xanthoxylin & N.D. & N.D. & N.D. & $36.9 \pm 2.3^{a}$ & $26.5 \pm 3.5^{b}$ & $39.8 \pm 5.4^{\mathrm{a}}$ & $26.6 \pm 2.2^{b}$ & 0.003 & VFAB \\
\hline & Hydrocarbons (25) & $280.1 \pm 26.5^{f}$ & $2357.7 \pm 80.2^{\mathrm{a}}$ & $1680.4 \pm 41.0^{\mathrm{e}}$ & $1946.6 \pm 146.6^{\mathrm{d}}$ & $2227.3 \pm 43.0^{\mathrm{b}}$ & $2087.4 \pm 43.4^{c}$ & $2295.6 \pm 30.1^{\mathrm{ab}}$ & 0.000 & \\
\hline 73 & 2-Methylbutane & N.D. & $2.9 \pm 0.6^{a}$ & N.D. & $1.6 \pm 0.2^{b}$ & N.D. & $3.1 \pm 0.2^{\mathrm{a}}$ & $2.7 \pm 0.3^{a}$ & 0.005 & LOP \\
\hline 74 & Benzene & N.D. & N.D. & $5.4 \pm 0.7^{\mathrm{a}}$ & $2.8 \pm 0.1^{b}$ & N.D. & N.D. & N.D. & 0.002 & LOP \\
\hline 75 & Toluene & $10.3 \pm 1.5^{\mathrm{e}}$ & $179.8 \pm 8.4^{b}$ & $238.8 \pm 23.4^{\mathrm{a}}$ & $119.9 \pm 14.6^{\mathrm{d}}$ & $141.4 \pm 9.9^{\mathrm{cd}}$ & $226.7 \pm 15.0^{a}$ & $163.3 \pm 8.8^{\mathrm{bc}}$ & 0.000 & AADP \\
\hline 76 & Ethylbenzene & $27.3 \pm 0.8^{b}$ & $11.3 \pm 0.4^{\mathrm{d}}$ & $16.3 \pm 1.5^{c}$ & $48.3 \pm 5.9^{a}$ & $5.2 \pm 1.8^{\mathrm{e}}$ & $1.2 \pm 0.2^{\mathrm{e}}$ & $3.6 \pm 0.06^{\mathrm{e}}$ & 0.000 & AADP \\
\hline 77 & 1,3-Dimethylbenzene & $22.2 \pm 2.0^{\mathrm{d}}$ & $160.0 \pm 7.1^{b}$ & $140.2 \pm 30.3^{b}$ & $148.7 \pm 15.1^{b}$ & $295.2 \pm 9.6^{a}$ & $86.2 \pm 13.8^{c}$ & $149.1 \pm 10.6^{\mathrm{b}}$ & 0.000 & AADP \\
\hline 78 & p-Xylene & N.D. & $270.1 \pm 4.8^{\mathrm{d}}$ & $367.3 \pm 10.0^{c}$ & $483.5 \pm 80.8^{b}$ & $525.5 \pm 21.4^{\mathrm{b}}$ & $618.1 \pm 10.2^{\mathrm{a}}$ & $622.4 \pm 18.3^{\mathrm{a}}$ & 0.000 & LOP \\
\hline 80 & D-Limonene & N.D. & $12.5 \pm 1.8^{c}$ & $22.0 \pm 0.7^{b}$ & $13.2 \pm 1.7^{\mathrm{c}}$ & $21.8 \pm 2.4^{b}$ & $29.9 \pm 3.1^{\mathrm{a}}$ & $13.3 \pm 0.7^{c}$ & 0.000 & VFAB \\
\hline 81 & Phellandrene & N.D. & N.D. & N.D. & $3.4 \pm 1.1^{\mathrm{a}}$ & $2.3 \pm 0.2^{a}$ & N.D. & N.D. & 0.218 & VFAB \\
\hline 82 & $\begin{array}{l}\text { (Z)-3,7-Dimethyl-1,3,6- } \\
\text { octatriene }\end{array}$ & N.D. & N.D. & N.D. & $10.9 \pm 0.8^{b}$ & N.D. & $13.4 \pm 0.8^{\mathrm{a}}$ & N.D. & 0.019 & VFAB \\
\hline 83 & $\begin{array}{c}1,3,5,7- \\
\text { Cyclooctatetraene }\end{array}$ & N.D. & $456.8 \pm 15.0^{\mathrm{d}}$ & $435.3 \pm 17.3^{\mathrm{d}}$ & $445.6 \pm 11.5^{\mathrm{d}}$ & $621.5 \pm 16.7^{\mathrm{a}}$ & $567.1 \pm 22.7^{b}$ & $530.5 \pm 17.1^{\mathrm{c}}$ & 0.000 & LOP \\
\hline 84 & Styrene & $45.0 \pm 6.3^{d}$ & $151.2 \pm 17.9^{b}$ & $88.2 \pm 11.2^{\mathrm{cd}}$ & $323.8 \pm 72.7^{\mathrm{a}}$ & $328.1 \pm 32.5^{\mathrm{a}}$ & $113.3 \pm 9.2^{b c}$ & $104.8 \pm 7.9^{b c}$ & 0.000 & VFAB \\
\hline 85 & o-Cymene & N.D. & N.D. & N.D. & $27.3 \pm 2.0^{a}$ & $20.6 \pm 4.4^{a}$ & N.D. & N.D. & 0.101 & VFAB \\
\hline 86 & 1,2,4-Trimethylbenzene & N.D. & $10.5 \pm 0.5^{\mathrm{a}}$ & $10.9 \pm 1.0^{\mathrm{a}}$ & $8.4 \pm 0.7^{b}$ & N.D. & $7.5 \pm 1.4^{b}$ & N.D. & 0.007 & LOP \\
\hline 87 & m-Cymene & N.D. & N.D. & N.D. & $0.4 \pm 0.1^{\mathrm{d}}$ & $77.2 \pm 4.0^{\mathrm{c}}$ & $93.6 \pm 7.5^{b}$ & $128.9 \pm 13.4^{\mathrm{a}}$ & 0.000 & VFAB \\
\hline 88 & 1,3,8-p-Menthatriene & N.D. & $22.9 \pm 2.2^{a}$ & $6.2 \pm 0.5^{c}$ & $6.2 \pm 1.0^{c}$ & N.D. & $9.2 \pm 1.0^{b}$ & $7.9 \pm 0.8^{b c}$ & 0.000 & LOP \\
\hline 89 & 1-Methylindan & N.D. & N.D. & N.D. & $7.5 \pm 0.4^{a}$ & $7.7 \pm 0.3^{a}$ & $6.9 \pm 0.3^{a}$ & $7.5 \pm 1.7^{\mathrm{a}}$ & 0.123 & VFAB \\
\hline
\end{tabular}


Table 2. Cont.

\begin{tabular}{|c|c|c|c|c|c|c|c|c|c|c|}
\hline No. & Compounds & FP & $\mathrm{SP}_{1}$ & $\mathrm{SP}_{2}$ & $\mathrm{SP}_{3}$ & $\mathrm{SP}_{4}$ & $\mathrm{SP}_{5}$ & $\mathrm{SP}_{6}$ & $p$ Value & Origin $^{1}$ \\
\hline 90 & Naphthalene & $3.5 \pm 0.4^{\mathrm{c}}$ & N.D. & $6.8 \pm 0.4^{b}$ & $7.8 \pm 1.1^{\mathrm{ab}}$ & $7.2 \pm 0.5^{\mathrm{ab}}$ & $7.9 \pm 0.4^{\mathrm{ab}}$ & $8.2 \pm 0.7^{\mathrm{a}}$ & 0.000 & UO \\
\hline 91 & Decane & N.D. & $6.4 \pm 0.3^{b c}$ & $9.3 \pm 0.2^{a}$ & $7.2 \pm 0.7^{b c}$ & $7.5 \pm 1.3^{b}$ & $5.9 \pm 1.0^{c}$ & $6.3 \pm 0.3^{b c}$ & 0.002 & LOP \\
\hline 93 & Tridecane & N.D. & $20.4 \pm 1.6^{a}$ & $16.2 \pm 2.1^{b}$ & $7.4 \pm 1.2^{\mathrm{d}}$ & $9.6 \pm 0.2^{c}$ & $5.9 \pm 0.1^{\mathrm{d}}$ & $6.3 \pm 0.1^{\mathrm{d}}$ & 0.000 & LOP \\
\hline 94 & Tetradecane & N.D. & N.D. & $21.4 \pm 1.0^{a}$ & N.D. & $12.9 \pm 1.3^{b}$ & $9.3 \pm 0.4^{c}$ & $14.0 \pm 0.6^{b}$ & 0.000 & LOP \\
\hline 95 & $\begin{array}{c}1,2,4,5- \\
\text { Tetramethylbenzene }\end{array}$ & $3.5 \pm 0.4^{\mathrm{e}}$ & $24.4 \pm 3.2^{\mathrm{ab}}$ & $27.0 \pm 1.6^{\mathrm{a}}$ & N.D. & $21.7 \pm 1.8^{b}$ & $8.4 \pm 0.2^{\mathrm{d}}$ & $17.4 \pm 1.2^{\mathrm{c}}$ & 0.000 & $\mathrm{UO}$ \\
\hline 96 & Pentadecane & N.D. & $9.3 \pm 0.3^{b}$ & $12.7 \pm 0.9^{a}$ & N.D. & N.D. & N.D. & N.D. & 0.003 & LOP \\
\hline \multirow[t]{2}{*}{97} & Longifolene & N.D. & N.D. & N.D. & N.D. & $2.6 \pm 0.05^{b}$ & $3.5 \pm 1.4^{b}$ & $5.8 \pm 0.6^{\mathrm{a}}$ & 0.011 & VFAB \\
\hline & Esters (13) & $1.9 \pm 0.3^{f}$ & $222.8 \pm 19.4^{\mathrm{c}}$ & $440.8 \pm 24.6^{a}$ & $120.8 \pm 14.0^{\mathrm{e}}$ & $166.2 \pm 12.6^{\mathrm{d}}$ & $399.9 \pm 12.9^{b}$ & $205.8 \pm 12.0^{\mathrm{c}}$ & 0.000 & \\
\hline 98 & Ethyl acetate & N.D. & $46.7 \pm 3.9^{c}$ & $69.9 \pm 3.8^{a}$ & $35.6 \pm 7.3^{\mathrm{d}}$ & $58.8 \pm 1.2^{b}$ & $34.6 \pm 4.2^{\mathrm{d}}$ & $47.8 \pm 1.0^{c}$ & 0.000 & LOP \\
\hline 99 & Ethenyl acetate & N.D. & $60.9 \pm 7.6^{c}$ & $210.3 \pm 21.3^{b}$ & $26.8 \pm 3.1^{d}$ & $2.4 \pm 0.8^{\mathrm{e}}$ & $247.0 \pm 11.9^{a}$ & $27.0 \pm 2.7^{d}$ & 0.000 & LOP \\
\hline 100 & Butyl butanoate & N.D. & $8.2 \pm 0.6^{c}$ & $12.3 \pm 0.5^{b}$ & $3.3 \pm 0.6^{\mathrm{d}}$ & N.D. & $15.0 \pm 1.2^{\mathrm{a}}$ & $14.2 \pm 1.2^{\mathrm{a}}$ & 0.022 & LOP \\
\hline 102 & Hexyl acetate & N.D. & N.D. & $3.7 \pm 0.3^{b}$ & N.D. & $2.5 \pm 0.2^{a}$ & N.D. & N.D. & 0.004 & $\mathrm{UO}$ \\
\hline 103 & Hexyl butanoate & N.D. & $2.4 \pm 0.3^{b}$ & $3.7 \pm 0.2^{a}$ & $0.9 \pm 0.2^{\mathrm{d}}$ & $1.5 \pm 0.4^{\mathrm{c}}$ & $1.4 \pm 0.3^{c}$ & $3.2 \pm 0.2^{a}$ & 0.000 & LOP \\
\hline 104 & $\begin{array}{l}\text { 1-(Acetyloxy)-2- } \\
\text { propanone }\end{array}$ & N.D. & $40.9 \pm 2.7^{b}$ & $52.2 \pm 7.0^{a}$ & $25.2 \pm 1.4^{\mathrm{c}}$ & $23.9 \pm 2.3^{c}$ & $23.8 \pm 1.9^{c}$ & $29.8 \pm 3.2^{c}$ & 0.000 & LOP \\
\hline 105 & Butyrolactone & $1.9 \pm 0.3^{\mathrm{d}}$ & $2.1 \pm 0.5^{b c}$ & $3.9 \pm 0.6^{a}$ & $1.7 \pm 0.2^{\mathrm{d}}$ & $2.6 \pm 0.5^{b}$ & $2.1 \pm 0.2^{b c}$ & $2.4 \pm 0.1^{b}$ & 0.000 & LOP \\
\hline 106 & $\begin{array}{l}\text { 5-Ethyldihydro-2(3H)- } \\
\text { furanone }\end{array}$ & N.D. & $1.8 \pm 0.4^{b}$ & $2.0 \pm 0.3^{a}$ & N.D. & $0.5 \pm 0.1^{\mathrm{c}}$ & $0.6 \pm 0.01^{c}$ & $0.5 \pm 0.1^{\mathrm{c}}$ & 0.000 & LOP \\
\hline 107 & Hexanolactone & N.D. & N.D. & $0.1 \pm 0.01^{b}$ & $0.8 \pm 0.2^{a}$ & N.D. & N.D. & $0.9 \pm 0.1^{a}$ & 0.000 & LOP \\
\hline 108 & Phenethyl acetate & N.D. & N.D. & N.D. & $1.9 \pm 0.1^{\mathrm{a}}$ & $0.8 \pm 0.2^{b}$ & $0.8 \pm 0.2^{b}$ & $0.6 \pm 0.1^{b}$ & 0.000 & VFAB \\
\hline 109 & Eugenol acetate & N.D. & N.D. & N.D. & $3.0 \pm 0.5^{b}$ & $3.7 \pm 0.6^{b}$ & $5.1 \pm 0.5^{a}$ & $1.3 \pm 0.1^{\mathrm{c}}$ & 0.000 & VFAB \\
\hline 110 & Coumarin & N.D. & N.D. & N.D. & $0.2 \pm 0.01^{b}$ & N.D. & $0.3 \pm 0.01^{a}$ & N.D. & 0.041 & VFAB \\
\hline
\end{tabular}


Table 2. Cont.

\begin{tabular}{|c|c|c|c|c|c|c|c|c|c|c|}
\hline No. & Compounds & FP & $\mathrm{SP}_{1}$ & $\mathrm{SP}_{2}$ & $\mathrm{SP}_{3}$ & $\mathrm{SP}_{4}$ & $\mathrm{SP}_{5}$ & $\mathrm{SP}_{6}$ & $p$ Value & Origin $^{1}$ \\
\hline & Ethers (5) & $2.8 \pm 0.5^{\mathrm{f}}$ & $16.5 \pm 0.5^{\mathrm{e}}$ & $16.9 \pm 2.1^{\mathrm{e}}$ & $116.8 \pm 3.2^{\mathrm{a}}$ & $37.1 \pm 2.8^{c}$ & $41.7 \pm 2.2^{b}$ & $25.0 \pm 2.0^{d}$ & 0.000 & \\
\hline 111 & Anethole & $2.8 \pm 0.5^{\mathrm{e}}$ & $16.5 \pm 0.5^{d}$ & $16.6 \pm 2.1^{d}$ & $80.7 \pm 1.7^{a}$ & $33.3 \pm 2.9^{b}$ & $35.1 \pm 1.8^{b}$ & $21.8 \pm 1.8^{c}$ & 0.000 & VFAB \\
\hline 113 & Methyleugenol & N.D. & N.D. & N.D. & $2.0 \pm 0.1^{a}$ & $0.9 \pm 0.2^{c}$ & $1.3 \pm 0.1^{b}$ & $0.4 \pm 0.01^{\mathrm{d}}$ & 0.000 & VFAB \\
\hline 114 & Elemicin & N.D. & N.D. & N.D. & $1.1 \pm 0.2^{\mathrm{a}}$ & $0.6 \pm 0.1^{b}$ & $1.3 \pm 0.1^{\mathrm{a}}$ & $0.4 \pm 0.01^{b}$ & 0.000 & VFAB \\
\hline \multirow[t]{2}{*}{115} & Myristicin & N.D. & N.D. & N.D. & $1.5 \pm 0.2^{\mathrm{a}}$ & $0.8 \pm 0.1^{c}$ & $1.1 \pm 0.04^{b}$ & $0.3 \pm 0.02^{d}$ & 0.000 & VFAB \\
\hline & Phenols (3) & $0.2 \pm 0.01^{\mathrm{e}}$ & N.D. & N.D. & $25.4 \pm 2.3^{b}$ & $21.0 \pm 0.6^{\mathrm{c}}$ & $31.7 \pm 4.5^{\mathrm{a}}$ & $10.6 \pm 0.3^{d}$ & 0.000 & \\
\hline 116 & Phenol & $0.2 \pm 0.01^{\mathrm{a}}$ & N.D. & N.D. & $0.2 \pm 0.02^{a}$ & $0.2 \pm 0.02^{a}$ & $0.2 \pm 0.01^{\mathrm{a}}$ & $0.2 \pm 0.02^{a}$ & 0.121 & $\mathrm{UO}$ \\
\hline 117 & Eugenol & N.D. & N.D. & N.D. & $21.6 \pm 2.8^{b}$ & $18.4 \pm 0.7^{b}$ & $28.9 \pm 4.5^{\mathrm{a}}$ & $9.2 \pm 0.5^{c}$ & 0.000 & VFAB \\
\hline \multirow[t]{2}{*}{118} & trans-Isoeugenol & N.D. & N.D. & N.D. & $3.6 \pm 0.6^{a}$ & $2.4 \pm 0.2^{b}$ & $2.6 \pm 0.05^{b}$ & $1.8 \pm 0.3^{c}$ & 0.000 & VFAB \\
\hline & Acids (4) & $45.2 \pm 3.2^{a}$ & $23.0 \pm 1.3^{b c}$ & $45.0 \pm 2.9^{a}$ & $19.9 \pm 1.4^{\mathrm{c}}$ & $13.6 \pm 0.9^{d}$ & $24.9 \pm 2.1^{b}$ & $24.2 \pm 0.6^{b}$ & 0.000 & \\
\hline 119 & Acetic acid & N.D. & N.D. & $15.6 \pm 1.4^{b}$ & $14.7 \pm 1.7^{b}$ & $6.9 \pm 0.5^{c}$ & $18.6 \pm 1.9^{a}$ & $16.6 \pm 1.4^{\mathrm{ab}}$ & 0.000 & $\mathrm{UO}$ \\
\hline 121 & Pentanoic acid & $32.1 \pm 2.0^{\mathrm{a}}$ & N.D. & $29.4 \pm 2.0^{a}$ & $5.2 \pm 0.3^{c}$ & $6.7 \pm 0.7^{b}$ & $6.3 \pm 0.4^{b}$ & $7.6 \pm 1.2^{b}$ & 0.000 & VFRM \\
\hline \multirow[t]{2}{*}{122} & Octanoic acid & $2.6 \pm 0.3$ & N.D. & N.D. & N.D. & N.D. & N.D. & N.D. & N.D. & VFRM \\
\hline & $\begin{array}{c}\text { Heterocyclic and } \\
\text { sulfur compounds (17) }\end{array}$ & $2.4 \pm 0.2^{c}$ & $1192.7 \pm 104.0^{\mathrm{a}}$ & $1099.0 \pm 198.8^{\mathrm{a}}$ & $312.4 \pm 32.1^{b}$ & $331.9 \pm 17.2^{b}$ & $331.8 \pm 2.3^{b}$ & $330.8 \pm 19.7^{b}$ & 0.000 & \\
\hline 123 & 2-Pentylfuran & N.D. & $1071.5 \pm 108.9^{a}$ & $\begin{array}{c}975.5 \pm 178.1 \\
85 \mathrm{a}\end{array}$ & $249.0 \pm 28.0^{b}$ & $250.1 \pm 21.7^{b}$ & $234.3 \pm 3.1^{b}$ & $234.2 \pm 15.9^{b}$ & 0.001 & LOP \\
\hline 124 & $\begin{array}{l}\text { 3-(4-Methyl-3- } \\
\text { pentenyl)-furan }\end{array}$ & N.D. & N.D. & N.D. & $4.7 \pm 1.0^{\mathrm{b}}$ & $3.6 \pm 0.03^{c}$ & $5.8 \pm 0.1^{a}$ & $1.7 \pm 0.1^{\mathrm{d}}$ & 0.000 & MRP \\
\hline 125 & Furfural & N.D. & N.D. & $1.9 \pm 0.4^{b}$ & $2.1 \pm 0.3^{b}$ & $2.9 \pm 0.2^{a}$ & $3.0 \pm 0.7^{a}$ & $3.4 \pm 0.2^{a}$ & 0.005 & MRP \\
\hline 126 & 2-Furanmethanol & N.D. & N.D. & N.D. & $0.2 \pm 0.1^{\mathrm{c}}$ & $0.5 \pm 0.1^{b}$ & $0.7 \pm 0.02^{a}$ & $0.5 \pm 0.1^{b}$ & 0.000 & MRP \\
\hline 127 & $\begin{array}{c}5- \\
\text { Hydroxymethylfurfural }\end{array}$ & N.D. & $57.0 \pm 6.8^{a}$ & $20.0 \pm 0.7^{b}$ & N.D. & N.D. & N.D. & N.D. & 0.011 & MRP \\
\hline 128 & Safrole & N.D. & N.D. & N.D. & $0.9 \pm 0.2^{a}$ & $0.6 \pm 0.1^{b}$ & $0.5 \pm 0.03^{b}$ & $0.4 \pm 0.1^{b}$ & 0.062 & VFAB \\
\hline 129 & Pyridine & $0.9 \pm 0.1^{c}$ & $1.5 \pm 0.3^{b}$ & $0.8 \pm 0.1^{\mathrm{d}}$ & $1.4 \pm 0.03^{b}$ & $0.8 \pm 0.3^{d}$ & $1.1 \pm 0.1^{\mathrm{c}}$ & $2.7 \pm 0.2^{a}$ & 0.000 & MRP \\
\hline
\end{tabular}


Table 2. Cont.

\begin{tabular}{|c|c|c|c|c|c|c|c|c|c|c|}
\hline No. & Compounds & FP & $\mathrm{SP}_{1}$ & $\mathrm{SP}_{2}$ & $\mathrm{SP}_{3}$ & $\mathrm{SP}_{4}$ & $\mathrm{SP}_{5}$ & $\mathrm{SP}_{6}$ & $p$ Value & Origin $^{1}$ \\
\hline 130 & 2-Propylpyridine & N.D. & N.D. & $2.9 \pm 1.0$ & N.D. & N.D. & N.D. & N.D. & N.D. & MRP \\
\hline 131 & 2-Acetylpyrrole & N.D. & N.D. & N.D. & $0.1 \pm 0.01^{\mathrm{c}}$ & $0.5 \pm 0.2^{b}$ & $1.3 \pm 0.1^{\mathrm{a}}$ & $1.5 \pm 0.3^{a}$ & 0.001 & MRP \\
\hline 132 & Methanethiol & $1.0 \pm 0.3^{\mathrm{e}}$ & $47.5 \pm 3.7^{c}$ & $84.1 \pm 19.0^{a}$ & $32.9 \pm 2.5^{d}$ & $51.1 \pm 4.1^{\mathrm{c}}$ & $68.5 \pm 1.5^{b}$ & $56.4 \pm 2.6^{b}$ & 0.000 & AADP \\
\hline 133 & Dimethyl disulfide & N.D. & $0.9 \pm 0.1^{b}$ & $5.5 \pm 0.5^{a}$ & N.D. & N.D. & N.D. & N.D. & 0.000 & AADP \\
\hline 134 & 3-Methylthiophene & N.D. & $12.5 \pm 1.0^{\mathrm{c}}$ & $7.2 \pm 1.3^{\mathrm{d}}$ & $19.5 \pm 2.7^{b}$ & $17.7 \pm 1.0^{\mathrm{b}}$ & $13.0 \pm 1.5^{\mathrm{c}}$ & $26.3 \pm 1.0^{a}$ & 0.000 & AADP \\
\hline 135 & Dimethyl sulfone & $0.5 \pm 0.01^{b}$ & N.D. & $0.6 \pm 0.02^{a}$ & $0.5 \pm 0.1^{b}$ & $0.3 \pm 0.02^{c}$ & $0.3 \pm 0.01^{\mathrm{c}}$ & $0.5 \pm 0.1^{b}$ & 0.001 & AADP \\
\hline 136 & Dimethyl trisulfide & N.D. & $1.4 \pm 0.1^{\mathrm{b}}$ & N.D. & N.D. & $1.5 \pm 0.2^{b}$ & $2.1 \pm 0.1^{a}$ & $1.6 \pm 0.2^{b}$ & 0.001 & MRP \\
\hline 137 & 2-Acetylthiazole & N.D. & N.D. & N.D. & $0.9 \pm 0.1^{\mathrm{c}}$ & $2.0 \pm 0.1^{a}$ & $0.9 \pm 0.3^{c}$ & $1.4 \pm 0.1^{b}$ & 0.000 & MRP \\
\hline 138 & $\begin{array}{c}2- \\
\text { Thiophenecarboxaldehyde }\end{array}$ & N.D. & N.D. & $0.2 \pm 0.02^{\mathrm{a}}$ & $0.2 \pm 0.01^{\mathrm{a}}$ & N.D. & N.D. & N.D. & 0.786 & $\mathrm{MRP}$ \\
\hline \multirow[t]{2}{*}{139} & Benzothiazole & N.D. & $0.4 \pm 0.1^{a}$ & $0.3 \pm 0.01^{b}$ & N.D. & $0.3 \pm 0.02^{b}$ & $0.3 \pm 0.01^{b}$ & $0.2 \pm 0.02^{b}$ & 0.067 & MRP \\
\hline & Total & $1669.8 \pm 97.1^{\mathrm{c}}$ & $8096.5 \pm 247.1^{a}$ & $8546.0 \pm 623.7^{a}$ & $4788.4 \pm 201.5^{b}$ & $4447.1 \pm 87.1^{b}$ & $4414.2 \pm 49.5^{b}$ & $4692.3 \pm 58.2^{b}$ & 0.000 & \\
\hline
\end{tabular}

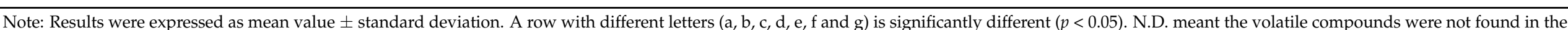

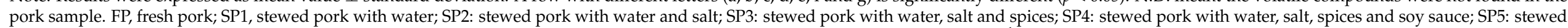

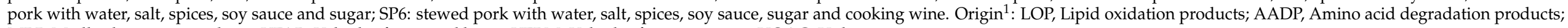
MRP, Maillard reaction products; VFAB, Volatiles from aged brine; VFRM, Volatiles from raw meat, UOAC, Unknown origin. 
Ester compounds could be formed by the esterification of alcohols and carboxylic acids in the meat products [2]. The contribution of esters to the aroma of pork products depends on the length of their chain [27]. A total of 13 esters were identified in all stewed pork samples, where short-chain esters, such as ethyl acetate and ethenyl acetate, had fruity notes. While long-chain esters like isoamyl isobutyrate, hexyl butanoate, hexyl acetate and hexyl butanoate possessed a slight fatty odour [36]. In addition, when the salt was added, the relative content of esters was significantly increased $(p<0.05)$. This reason might be that the salt of meat products favoured the formation of ester compounds. For ether and phenol compounds, the anethole was detected in all pork samples and the methyleugenol, elemicin, myristicin, eugenol and trans-isoeugenol were found in $\mathrm{SP}_{3}, \mathrm{SP}_{4}, \mathrm{SP}_{5}$ and $\mathrm{SP}_{6}$. Moreover, the ethers and phenols except for phenol were from the spices, and the acids (butanoic acid, pentanoic acid, and octanoic acid) come from the fresh pork.

Heterocyclic and sulphur-containing compounds are the important contributions to the formation of flavour in meat products [37]. As shown in Table 2, 17 heterocyclic compounds (furans, pyridines and pyrroles) and sulphur-containing compounds were detected in all pork samples. The 2-pentylfuran and safrole were derived from linoleic acid autoxidation [38] and spices (nutmeg, anise and ginger), respectively. The 2-pentylfuran is often used as an important indicator of the degree of oxidation of meat product. The contents of 2-pentylfuran in $\mathrm{SP}_{1}$ and $\mathrm{SP}_{2}$ were significantly higher $(p<0.001)$ than that in $\mathrm{SP}_{3}, \mathrm{SP}_{4}$, $\mathrm{SP}_{5}$, and $\mathrm{SP}_{6}$, indicating that the stewed pork with only water and salt had a greater effect on lipid oxidation. It has been reported that a large number of furans, pyridines, pyrroles and sulphur-containing compounds could be produced by Maillard reaction and amino acid degradation during cooking [15,23]. In our study, 3-(4-methyl-3-pentenyl)-furan, furfural, 2-furanmethanol, pyridine, 2-acetylpyrrole and dimethyl trisulfide displayed significantly higher levels $(p<0.01)$ in $\mathrm{SP}_{5}$ and $\mathrm{SP}_{6}$ than those in other groups, which indicated that the addition of sugar and cooking wine could promote the Maillard reaction. For sulphur-containing compounds, methanethiol and dimethyl disulphide from sulphurcontaining amino acid degradation were significantly lower $(p<0.001)$ in $\mathrm{SP}_{1}$ than that in $\mathrm{SP}_{2}$, which indicated that salt-treated stewed pork was more conducive to the production of sulphur-containing compounds. This result was consistent with that reported by Liu et al. [39] who found that the levels of sulphur-containing compounds in Nanjing water-boiled salted duck were markedly higher than those in control samples. Regarding 2-acetylthiazole, 2-thiophenecarboxaldehyde and benzothiazole originated from Maillard reaction, and they contributed to roasted, caramel, and meaty notes [40] for the overall aroma of stewed pork.

The concentration of volatile compounds according to possible origins of the fresh and stewed pork are presented in Figure 1. It was found that the lipid oxidation, aged brine and amino acid degradation were the important origins of volatile compounds in all stewed pork attributed to their contribution to more aroma of the pork samples. For the lipid oxidation and amino acid degradation, their concentrations were highest in $\mathrm{SP}_{1}$ and $\mathrm{SP}_{2}$, followed by $\mathrm{SP}_{3}, \mathrm{SP}_{4}, \mathrm{SP}_{5}$ and $\mathrm{SP}_{6}$, finally FP. This indicated that heat-treated pork with water and salt would facilitate lipid oxidation and amino acid degradation to produce more volatiles, while there was an inhibitory effect on heat-treated pork with aged brine, especially for spices. Compared with volatile compounds from the aged brine in $\mathrm{SP}_{3}, \mathrm{SP}_{4}$, $\mathrm{SP}_{5}$ and $\mathrm{SP}_{6}$, they were significantly higher $(p<0.05)$ than those in $\mathrm{SP}_{1}$ and $\mathrm{SP}_{2}$, this may be due to the addition of food condiments (spices, soy sauce, sugar, and cooking wine) in stewed pork.

\subsection{Odour-Active Compounds Analysis of the Fresh and Stewed Pork}

To evaluate the contributions of volatile compounds to overall flavour of the fresh and stewed pork, the OAVs of these compounds were determined by dividing the concentration of the compound by its odour threshold in water. As can be seen from Table 3, a total of 29 odour-active compounds with OAVs greater than 1 were selected from 139 volatile compounds, including 14 aldehydes, four alcohols, three ketones, one hydrocarbon, 
one ester, two ethers, one phenol, thre furans, $\mathrm{N}$ - or S-containing compounds. Seven of them with relatively high OAVs were detected in all stewed pork samples: hexanal (OAV at 44.1-158.5), heptanal (OAV at 10.5-53.4), octanal (OAV at 72.2-329.9), nonanal (OAV at 110.3-1475.5), oct-1-en-3-ol (OAV at 13.9-68.5), 2-pentylfuran (OAV at 39.0-178.6) and methanethiol (OAV at 31.3-80.1). These compounds were known as the key odour-active compounds due to their significant contributions to the integral flavour. Furthermore, it was found that the OAVs of hexanal, heptanal, octanal, nonanal, and decanal increased significantly $(p<0.01)$ in $\mathrm{SP}_{1}$ and $\mathrm{SP}_{2}$.

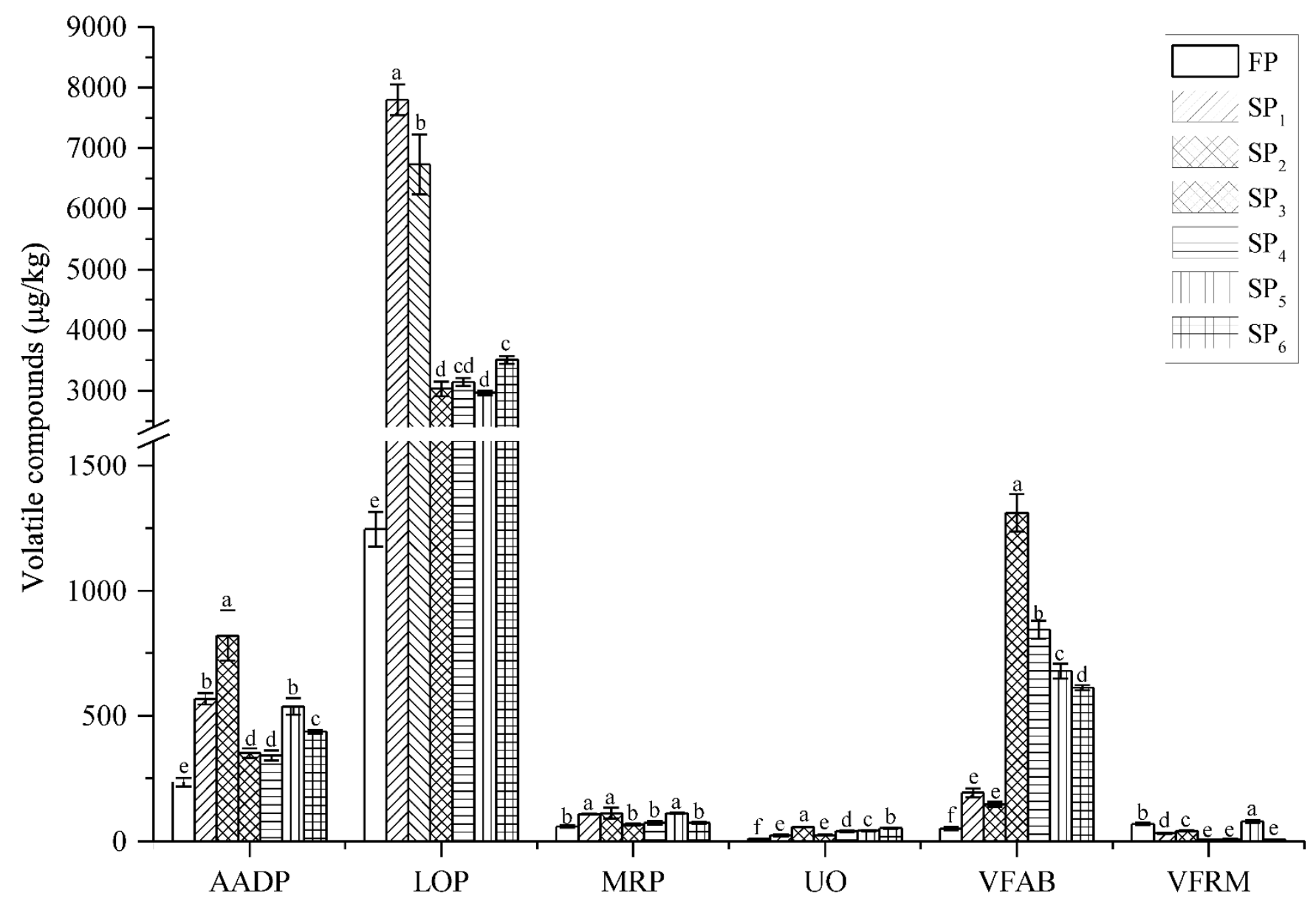

Figure 1. Concentration of volatile compounds according to possible origins of the fresh and stewed pork. Different letters are significantly different $(p<0.05)$ in each pork treatment group. $\mathrm{FP}$, fresh pork; $\mathrm{SP}_{1}$, boiled pork with water; $\mathrm{SP}_{2}$, cooked pork with water and salt; $\mathrm{SP}_{3}$, stewed pork with water, salt and spices; $\mathrm{SP}_{4}$, stewed pork with water, salt, spices and soy sauce; $\mathrm{SP}_{5}$, stewed pork with water, salt, spices, soy sauce and sugar; $\mathrm{SP}_{6}$, stewed pork with water, salt, spices, soy sauce, sugar and cooking wine. LOP, Lipid oxidation products; AADP, Amino acid degradation products; MRP, Maillard reaction products; VFAB, Volatiles from aged brine; VFRM, Volatiles from raw meat; UO, Unknown origin. 
Table 3. Odour-active compounds $(\mathrm{OAVs}>1)$ in the fresh and stewed pork.

\begin{tabular}{|c|c|c|c|c|c|c|c|c|c|c|}
\hline No. & Compounds & $\begin{array}{c}{ }^{1} \text { Odour } \\
\text { Descriptions; } \\
{ }^{2} \text { Odour Thresholds } \\
\left(\mu \mathrm{g} \cdot \mathrm{kg}^{-1}\right)\end{array}$ & FP & $\mathrm{SP}_{1}$ & $\mathrm{SP}_{2}$ & $\mathrm{SP}_{3}$ & $\mathrm{SP}_{4}$ & $\mathrm{SP}_{5}$ & $\mathrm{SP}_{6}$ & $p$ Value \\
\hline 4 & Pentanal & Almond, pungent; 9 & $3.1 \pm 0.4^{\mathrm{e}}$ & $6.0 \pm 0.8^{\mathrm{d}}$ & $8.3 \pm 0.1^{a b}$ & $9.0 \pm 0.4^{\mathrm{a}}$ & $6.7 \pm 1.1^{\mathrm{cd}}$ & $7.8 \pm 0.6^{\mathrm{bc}}$ & $7.3 \pm 0.4^{b c}$ & 0.000 \\
\hline 5 & Hexanal & Grass, fat; 4 & $0.9 \pm 0.3^{\mathrm{e}}$ & $158.3 \pm 3.3^{a}$ & $158.5 \pm 3.7^{\mathrm{a}}$ & $58.3 \pm 2.2^{c}$ & $63.5 \pm 2.5^{b}$ & $44.1 \pm 0.2^{\mathrm{d}}$ & $55.3 \pm 3.9^{c}$ & 0.000 \\
\hline 6 & Heptanal & Fat, citrus; 3 & $18.9 \pm 1.1^{c}$ & $40.9 \pm 5.3^{b}$ & $53.4 \pm 0.7^{\mathrm{a}}$ & $10.5 \pm 0.6^{\mathrm{d}}$ & $12.3 \pm 0.4^{\mathrm{d}}$ & $12.8 \pm 1.2^{\mathrm{d}}$ & $12.7 \pm 2.4^{\mathrm{d}}$ & 0.000 \\
\hline 7 & Octanal & Fat, lemon, green; 0.7 & $121.0 \pm 15.5^{c}$ & $260.0 \pm 14.8^{b}$ & $329.9 \pm 35.8^{a}$ & $89.2 \pm 7.1^{d}$ & $123.4 \pm 13.8^{c}$ & $72.2 \pm 6.1^{\mathrm{d}}$ & $78.5 \pm 12.3^{d}$ & 0.000 \\
\hline 8 & (Z)-Hept-2-enal & Fishy; 13.5 & N.D. & $0.4 \pm 0.01^{b}$ & $2.9 \pm 0.7^{a}$ & N.D. & N.D. & N.D. & N.D. & 0.024 \\
\hline 10 & Nonanal & Fat, citrus, green; 1 & $219.8 \pm 13.3^{\mathrm{cd}}$ & $844.4 \pm 94.8^{b}$ & $1475.5 \pm 124.6^{\mathrm{a}}$ & $316.3 \pm 5.8^{c}$ & $171.4 \pm 17.8^{\mathrm{d}}$ & $110.3 \pm 7.9^{d}$ & $284.2 \pm 11.9^{c}$ & 0.000 \\
\hline 11 & (E)-Oct-2-enal & Green, nut, fat; 3 & $8.2 \pm 0.4^{c}$ & $25.3 \pm 0.4^{\mathrm{a}}$ & $14.1 \pm 2.5^{b}$ & $1.1 \pm 0.1^{\mathrm{d}}$ & $1.8 \pm 0.4^{\mathrm{d}}$ & $2.2 \pm 0.6^{\mathrm{d}}$ & $2.8 \pm 0.2^{\mathrm{d}}$ & 0.000 \\
\hline 12 & Decanal & Soap, orange peel; 2 & $2.6 \pm 0.3^{b c}$ & $4.5 \pm 0.9^{\mathrm{a}}$ & $4.0 \pm 0.3^{a}$ & $2.2 \pm 0.1^{b c}$ & $2.3 \pm 0.3^{b c}$ & $2.1 \pm 0.1^{\mathrm{c}}$ & $3.0 \pm 0.2^{b}$ & 0.001 \\
\hline 14 & (E)-Non-2-enal & Cucumber, green; 0.19 & $39.2 \pm 5.0^{c}$ & $59.5 \pm 13.3^{b}$ & $79.5 \pm 5.2^{a}$ & $8.6 \pm 0.6^{\mathrm{d}}$ & $9.7 \pm 3.6^{d}$ & $12.6 \pm 1.9^{\mathrm{d}}$ & $10.0 \pm 2.4^{\mathrm{d}}$ & 0.000 \\
\hline 17 & (E)-Dec-2-enal & Orange; 0.3 & $9.8 \pm 0.7^{c}$ & $18.6 \pm 3.6^{a}$ & $15.4 \pm 3.4^{\mathrm{b}}$ & $3.3 \pm 0.5^{\mathrm{d}}$ & $2.0 \pm 0.1^{d}$ & $2.5 \pm 0.4^{d}$ & $2.7 \pm 0.2^{\mathrm{d}}$ & 0.000 \\
\hline 19 & $(E, E)-2,4-$ Nonadienal & $\begin{array}{c}\text { Geranium, pungent; } \\
0.09\end{array}$ & $30.1 \pm 3.0^{b}$ & $67.7 \pm 3.2^{a}$ & $64.0 \pm 2.5^{a}$ & N.D. & N.D. & N.D. & N.D. & 0.000 \\
\hline 20 & Undec-2-enal & Sweet; 0.78 & $3.3 \pm 0.3^{a}$ & $3.5 \pm 0.9^{a}$ & $3.1 \pm 0.4^{\mathrm{a}}$ & N.D. & N.D. & N.D. & N.D. & 0.717 \\
\hline 22 & $(E, E)$-2,4-Decadienal & Fried, wax, fat; 0.07 & $104.3 \pm 14.7^{b}$ & $143.3 \pm 7.6^{a}$ & $113.4 \pm 13.1^{\mathrm{b}}$ & $10.7 \pm 0.6^{c}$ & N.D. & $12.4 \pm 2.9^{c}$ & $22.7 \pm 1.9^{c}$ & 0.000 \\
\hline \multicolumn{11}{|c|}{ Alcohols (4) } \\
\hline 34 & 1,8-Cineole & Mint, sweet; 1 & $1.7 \pm 0.8^{\mathrm{e}}$ & $9.6 \pm 0.8^{\mathrm{d}}$ & $15.2 \pm 1.8^{\mathrm{d}}$ & $123.2 \pm 11.5^{\mathrm{a}}$ & $63.6 \pm 1.4^{\mathrm{b}}$ & $66.5 \pm 0.4^{b}$ & $43.0 \pm 1.1^{\mathrm{c}}$ & 0.000 \\
\hline 38 & Oct-1-en-3-ol & Mushroom; 2 & $0.7 \pm 0.2^{g}$ & $65.0 \pm 2.2^{b}$ & $68.5 \pm 1.5^{\mathrm{a}}$ & $25.3 \pm 0.3^{d}$ & $29.7 \pm 0.2^{c}$ & $13.9 \pm 1.0^{f}$ & $20.1 \pm 1.8^{\mathrm{e}}$ & 0.000 \\
\hline 41 & Linalool & Flower, lavender; 6 & N.D. & N.D. & N.D. & $12.6 \pm 1.0^{\mathrm{a}}$ & $3.8 \pm 0.3^{b}$ & $3.7 \pm 0.2^{b}$ & $3.6 \pm 0.4^{b}$ & 0.000 \\
\hline 45 & (E)-Oct-2-en-1-ol & Soap, plastic; 50 & $0.8 \pm 0.1^{c}$ & $1.5 \pm 0.2^{\mathrm{a}}$ & $1.2 \pm 0.2^{b}$ & $0.3 \pm 0.03^{\mathrm{d}}$ & $0.3 \pm 0.03^{d}$ & $0.3 \pm 0.04^{\mathrm{d}}$ & $0.3 \pm 0.01^{\mathrm{d}}$ & 0.000 \\
\hline \multicolumn{11}{|c|}{ Ketones (3) } \\
\hline 55 & Butane-2,3-dione & Butter; 4.37 & $2.9 \pm 0.5^{b c}$ & $5.4 \pm 0.4^{\mathrm{a}}$ & $5.3 \pm 0.7^{\mathrm{a}}$ & $5.6 \pm 0.5^{\mathrm{a}}$ & N.D. & $3.4 \pm 0.3^{b}$ & $2.5 \pm 0.1^{\mathrm{c}}$ & 0.000 \\
\hline
\end{tabular}


Table 3. Cont.

\begin{tabular}{|c|c|c|c|c|c|c|c|c|c|c|}
\hline No. & Compounds & $\begin{array}{c}{ }^{1} \text { Odour } \\
\text { Descriptions; } \\
{ }^{2} \text { Odour Thresholds } \\
\left(\mu \mathrm{g} \cdot \mathrm{kg}^{-1}\right)\end{array}$ & FP & $\mathrm{SP}_{1}$ & $\mathrm{SP}_{2}$ & $\mathrm{SP}_{3}$ & $\mathrm{SP}_{4}$ & $\mathrm{SP}_{5}$ & $\mathrm{SP}_{6}$ & $p$ Value \\
\hline \multirow[t]{2}{*}{61} & Octane-2,3-dione & Green, woody; 12 & $25.3 \pm 1.7^{c}$ & $48.2 \pm 2.2^{b}$ & $93.5 \pm 15.4^{a}$ & $26.8 \pm 0.8^{c}$ & $28.3 \pm 0.6^{c}$ & N.D. & $24.8 \pm 1.3^{c}$ & 0.000 \\
\hline & Hydrocarbon (1) & & & & & & & & & \\
\hline 80 & Ester (1) & & & & & & & & & \\
\hline \multirow[t]{2}{*}{98} & Ethyl acetate & Pineapple; 5 & N.D. & $9.3 \pm 0.8^{c}$ & $14.0 \pm 0.8^{\mathrm{a}}$ & $7.1 \pm 1.5^{\mathrm{d}}$ & $11.8 \pm 0.2^{b}$ & $6.9 \pm 0.8^{d}$ & $9.6 \pm 0.2^{c}$ & 0.000 \\
\hline & Ethers (2) & & & & & & & & & \\
\hline 111 & Anethole & Anissed-like; 15 & $0.2 \pm 0.03^{\mathrm{e}}$ & $1.1 \pm 0.04^{\mathrm{d}}$ & $1.1 \pm 0.1^{\mathrm{d}}$ & $5.4 \pm 0.1^{a}$ & $2.2 \pm 0.2^{b}$ & $2.3 \pm 0.1^{b}$ & $1.5 \pm 0.1^{\mathrm{c}}$ & 0.000 \\
\hline \multirow[t]{2}{*}{112} & Estragole & Licorice, anise; 6 & N.D. & N.D. & $0.1 \pm 0.02^{c}$ & $5.2 \pm 0.3^{a}$ & $0.3 \pm 0.03^{b c}$ & $0.5 \pm 0.1^{b}$ & $0.4 \pm 0.1^{b}$ & 0.000 \\
\hline & Phenol (1) & & & & & & & & & \\
\hline 117 & $\begin{array}{l}\text { Furan and sulfur } \\
\text { compounds ( } 3 \text { ) }\end{array}$ & & & & & & & & & \\
\hline 123 & 2-Pentylfuran & Green bean, butter; 6 & N.D. & $178.6 \pm 18.1^{a}$ & $162.6 \pm 29.7^{\mathrm{a}}$ & $41.5 \pm 4.7^{\mathrm{b}}$ & $41.7 \pm 3.6^{b}$ & $39.1 \pm 0.5^{b}$ & $39.0 \pm 2.4^{b}$ & 0.001 \\
\hline 132 & Methanethiol & $\begin{array}{l}\text { Sulfur, gasoline, } \\
\text { garlic; } 1.05\end{array}$ & $0.9 \pm 0.3^{\mathrm{e}}$ & $45.2 \pm 3.5^{c}$ & $80.1 \pm 18.1^{a}$ & $31.3 \pm 2.4^{\mathrm{d}}$ & $48.7 \pm 3.9^{c}$ & $65.3 \pm 1.5^{b}$ & $53.7 \pm 2.5^{b c}$ & 0.000 \\
\hline \multirow[t]{2}{*}{136} & Dimethyl trisulfide & $\begin{array}{c}\text { Sulfur, fish, cabbage; } \\
0.01\end{array}$ & N.D. & $136.7 \pm 9.1^{b}$ & N.D. & N.D. & $151.3 \pm 15.9^{b}$ & $212.4 \pm 12.5^{\mathrm{a}}$ & $163.4 \pm 17.2^{b}$ & 0.001 \\
\hline & Total & & $594.6 \pm 58.6^{d}$ & $2136.6 \pm 186.9^{b}$ & $2773.0 \pm 263.3^{a}$ & $801.7 \pm 42.0^{c}$ & $786.9 \pm 67.4^{c}$ & $710.1 \pm 43.1^{\mathrm{cd}}$ & $849.6 \pm 63.5^{c}$ & 0.000 \\
\hline
\end{tabular}

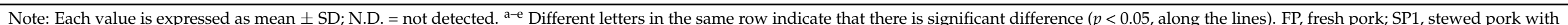

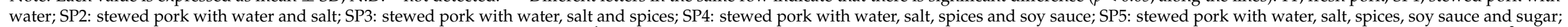

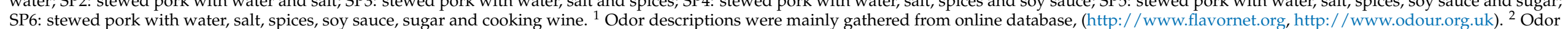
SP6: stewed pork with water, salt, spices, soy sauce, sugar and cooking wine. ${ }^{1}$ Odor descriptions were mainly gater.
thresholds were mainly obtained from online database, (http:// www.flavornet.org, http:// www.odour.org.uk). 
Statistical analysis showed that the total OAVs of odour-active compounds of $\mathrm{SP}_{1}$ and $\mathrm{SP}_{2}$ were significantly higher $(p<0.001)$ than those of $\mathrm{FP}$ and $\mathrm{SP}_{3}, \mathrm{SP}_{4}, \mathrm{SP}_{5}$ and $\mathrm{SP}_{6}$. Linear aldehydes like pentanal, hexanal, heptanal, octanal, nonanal and decanal have been reported to be generated from lipid oxidation [27]. Moreover, these aldehyde compounds could be detected in different processing methods and may contribute grassy, fatty and fruity notes to overall aroma of the pork samples. Unsaturated aldehydes such as (Z)-hept2-enal, $(E)$-oct-2-enal, $(E)$-non-2-enal, $(E)$-dec-2-enal, $(E, E)$-2,4-nonadienal, undec-2-enal, and $(E, E)-2,4$-decadienal are degradation products of linoleate and linolenate hydroperoxides [41]. Among them, there was no significant difference in undecen-2-al in FP, SP1, and SP2 $(p>0.05)$, indicating that heating and salt treatment had no effect on the formation of undecen-2-al. On the other hand, the rest of the olefin aldehydes have relatively higher $\mathrm{OAVs}$ in $\mathrm{SP}_{1}$ and $\mathrm{SP}_{2}$. This showed that $\mathrm{SP}_{1}$ and $\mathrm{SP}_{2}$ could promote the increase of some unsaturated aldehydes. Benzeneacetaldehyde, with honey and sweet notes, is a well-known aroma component formed from Maillard reaction of phenylalanine [42] and the $\mathrm{OAV}$ in $\mathrm{SP}_{5}$ were significantly higher $(p<0.001)$ than that in other samples. 1,8-Cineole, anethole and estragole, with mint and aniseed flavour, were the most abundant in $\mathrm{SP}_{3}$. The OAVs of oct-1-en-3-ol, (E)-oct-2-en-1-ol, butane-2,3-dione, octane-2,3-dione and 2-pentylfuran were the highest in $\mathrm{SP}_{1}$ and $\mathrm{SP}_{2}$, followed by $\mathrm{SP}_{3}, \mathrm{SP}_{4}, \mathrm{SP}_{5}$ and $\mathrm{SP}_{6}$, of which oct-1-en-3-ol and octane-2,3-dione was shown to be richer in $\mathrm{SP}_{2}$ than that in $\mathrm{SP}_{1}$. Linalool and eugenol were found immediately when the spices were added to the cooked pork, which may be due to the flavour of the star anise itself. Dimethyl trisulfide, with fish and cabbage notes, was considered as the main sulphur-compound in $\mathrm{SP}_{1}, \mathrm{SP}_{4}, \mathrm{SP}_{5}$, and $\mathrm{SP}_{6}$.

\subsection{PCA and PLS-DA Analysis of Odour-Active Compounds}

In order to clarify the differences in aroma profile of the fresh and stewed pork, a principal component analysis (PCA) was performed and showed in Figure 2a,b. The first principal component (PC1) explained $55.17 \%$, the second principal component (PC2) explained $22.85 \%$ and the third principal component (PC3) explained $13.22 \%$ of the variations. The first three principal components (PCs) accounted for $91.24 \%$ of the total variance and were enough to explain the maximum variation in all original data of the pork samples. As can be seen in Figure 2a, the PC1 and PC2 showed a clear-cut separation of the samples into three major groups. Among them, the sample dot of FP was located in the fourth quadrant, and sample dots representing $\mathrm{SP}_{3}, \mathrm{SP}_{4}, \mathrm{SP}_{5}$, and $\mathrm{SP}_{6}$ were located in the second quadrant and could be recognized as one cluster, while sample dots of $\mathrm{SP}_{1}$ and $\mathrm{SP}_{2}$ located in the first and fourth quadrant were considered as a group because of their relatively closer distance. As shown in Figure 2b, the fresh and stewed pork samples were divided into four groups in $\mathrm{PC} 1$ and $\mathrm{PC} 3$, that is, group I: FP; group II: $\mathrm{SP}_{1}$ and $\mathrm{SP}_{2}$; group III: $\mathrm{SP}_{3}$; group IV: $\mathrm{SP}_{4}, \mathrm{SP}_{5}$, and $\mathrm{SP}_{6}$. The four group of sample points were located in the different quadrants indicating that the overall aroma of each group of samples were different. Moreover, $\mathrm{SP}_{4}$, $\mathrm{SP}_{5}$, and $\mathrm{SP}_{6}$ samples was close and located in the third quadrant. It can be concluded that the overall flavour of $\mathrm{SP}_{4}, \mathrm{SP}_{5}$, and $\mathrm{SP}_{6}$ was similar each other. Similarly, so was $\mathrm{SP}_{1}$ and $\mathrm{SP}_{2}$. It can be also found that the flavour of samples $\mathrm{SP}_{4}, \mathrm{SP}_{5}$ and $\mathrm{SP}_{6}$ was significantly different from that of sample $\mathrm{SP}_{3}$ in Figure $2 \mathrm{a}, \mathrm{b}$. This showed that the flavour compounds of stewed pork were also affected by soy sauce, sugar, and cooking wine. 
(a) Observations (axes F1 and F2: 78.53\%)

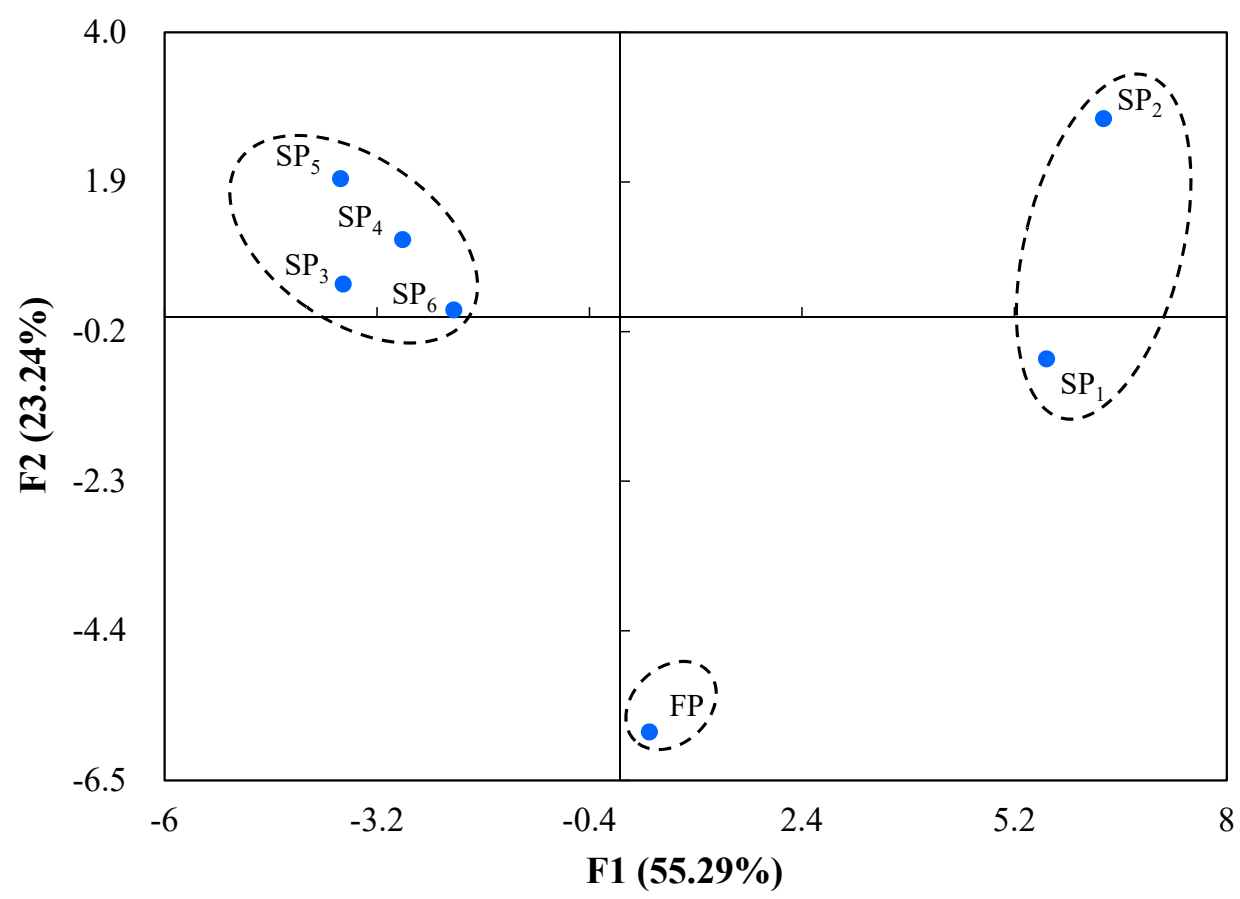

(b) Observations (axes F1 and F3: 68.53\%)

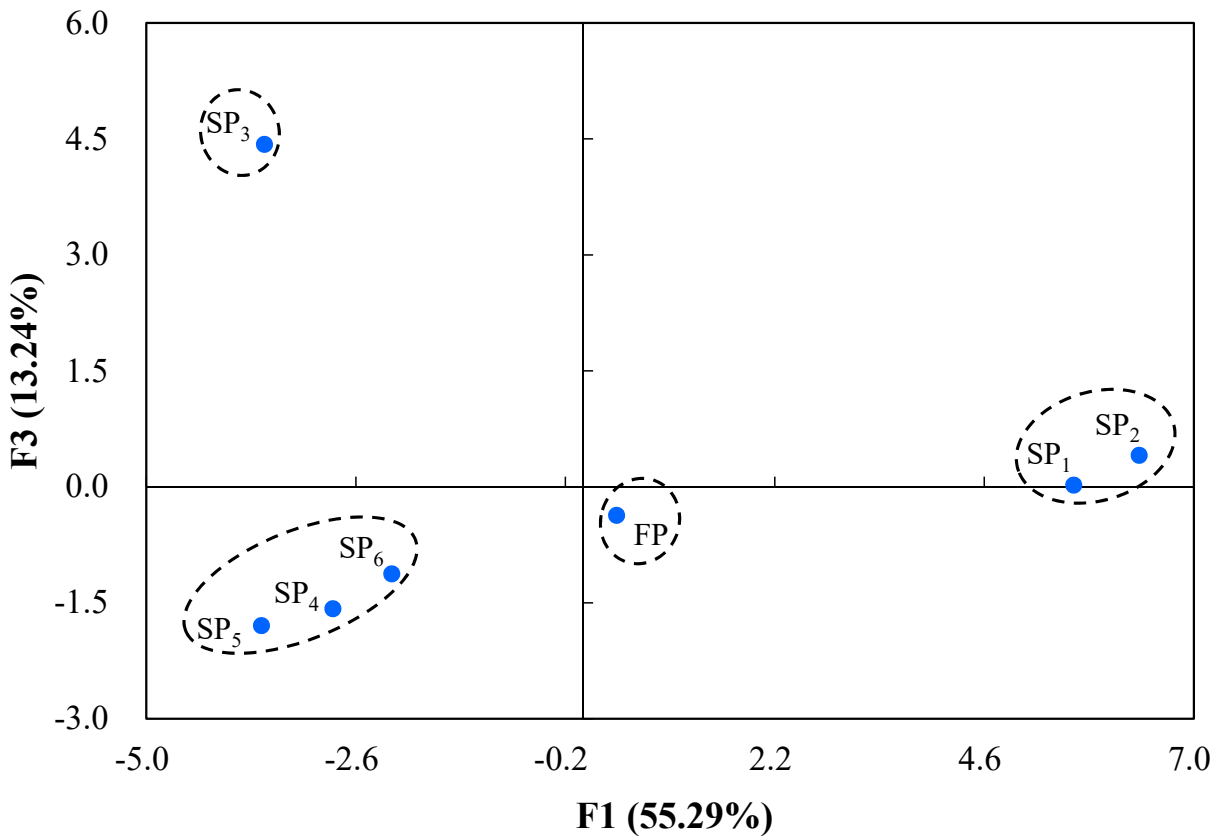

Figure 2. Score plots of PCA of the fresh and stewed pork. (a) PC1 plotted against PC2 and (b) PC1 against PC3. FP, fresh pork; $\mathrm{SP}_{1}$, stewed pork with water; $\mathrm{SP}_{2}$ : stewed pork with water and salt; $\mathrm{SP}_{3}$ : stewed pork with water, salt and spices; $\mathrm{SP}_{4}$ : stewed pork with water, salt, spices and soy sauce; $\mathrm{SP}_{5}$ : stewed pork with water, salt, spices, soy sauce and sugar; $\mathrm{SP}_{6}$ : stewed pork with water, salt, spices, soy sauce, sugar and cooking wine.

Apart from PCA, the supervised PLS-DA was performed to evaluated the differences of volatile compounds of the stewed pork. As shown in Figure 3a, except for $\mathrm{SP}_{3}, \mathrm{SP}_{4}, \mathrm{SP}_{5}$ and $\mathrm{SP}_{6}$, only the separation was observed for $\mathrm{FP}, \mathrm{SP}_{1}$, and $\mathrm{SP}_{2}\left(\mathrm{R}^{2} \mathrm{X}=0.968, \mathrm{R}^{2} \mathrm{Y}=0.818\right.$ and $\left.\mathrm{Q}^{2}=0.628\right) . \mathrm{SP}_{3}, \mathrm{SP}_{4}, \mathrm{SP}_{5}$, and $\mathrm{SP}_{6}$ were located on the negative side of axis 1 , whereas $\mathrm{FP}, \mathrm{SP}_{1}$, and $\mathrm{SP}_{2}$ were founds the positive side of axis1, $\mathrm{SP}_{1}$ and $\mathrm{SP}_{2}$ were close each other. Obviously, the different stewed pork samples were separated into three group $\left(\mathrm{SP}_{3}-\mathrm{SP}_{4}-\mathrm{SP}_{5}\right.$ 
$\mathrm{SP}_{6}, \mathrm{SP}_{1}-\mathrm{SP}_{2}, \mathrm{FP}$ ). It could also be concluded that the overall flavour of $\mathrm{SP}_{3}-\mathrm{SP}_{4}-\mathrm{SP}_{5}-\mathrm{SP}_{6}$, $\mathrm{SP}_{1}-\mathrm{SP}_{2}$ and $\mathrm{FP}$ were greatly different, and each group samples possessed the similar flavour profiles. The result is consistent with the PCA analysis (Figure 2a). In addition, to identify the most discriminative volatiles contributing to the observed the fresh and stewed pork samples, variable identification (VID) coefficients were calculated (Figure $3 b$ ). Volatiles with VID $\geq|0.80|$ discriminating $\mathrm{FP}, \mathrm{SP}_{1}, \mathrm{SP}_{2}$ and $\mathrm{SP}_{3}$ were predominantly aldehyde compounds (pentanal, (Z)-hept-2-enal, nonanal and (E)-oct-2-enal) and related volatiles (linalool, octane-2,3-dione, ethyl acetate, anethole and estragole). Moreover, no compound with VID $\geq 10.80 \mid$ was found discriminating $\mathrm{SP}_{4}, \mathrm{SP}_{5}$, and $\mathrm{SP}_{6}$. As shown in Figure 3a,b, it can be observed that pentanal, 1-hydroxypropan-2-one, D-limonene, ethyl acetate and methanethiol were not only on the opposite side of samples FP, but also strongly and negatively correlated with it $(-0.83 \leq \mathrm{r} \leq-0.69)$, while the remaining 24 odour-active compounds $(-0.57 \leq \mathrm{r} \leq 0.46)$ had a low correlation with it. Most odouractive compounds, such as hexanal, heptanal, octanal, $(Z)$-hept-2-enal, nonanal, (E)-oct2-enal, decanal, $(E)$-non-2-enal, $(E)$-dec-2-enal, $(E, E)$-2,4-nonadienal, $(E, E)$-2,4-decedienal, oct-1-en-3-ol, (E)-oct-2-en-1-ol, octane-2,3-dione, and 2-pentylfuran, were close to $\mathrm{SP}_{1}$ and $\mathrm{SP}_{2}$ on the right side of $\mathrm{t} 1$, and the strong and positive correlation $(0.60 \leq \mathrm{r} \leq 0.97)$ were showed. Moreover, $\mathrm{SP}_{2}$ induced an increase in the correlation coefficients of heptanal, octanal, (Z)-hept-2-enal, (E)-non-2-enal, oct-1-en-3-ol and octane-2,3-dione indicating that the addition of salt during the processing of stewed pork was beneficial to the formation of these compounds. For $\mathrm{SP}_{3}, \mathrm{SP}_{4}, \mathrm{SP}_{5}$ and $\mathrm{SP}_{6}$, benzeneacetaldehyde, 1,8-cineole, linalool, Dlimonene, anethole, estragole and eugenol had the high and positive correlation coefficients $(0.63 \leq \mathrm{r} \leq 0.99)$ on the left side of $\mathrm{t} 1$, only butane-2,3-dione had the high and negative correlation coefficient $(\mathrm{r}=-0.76)$. Because 1,8-cineole, linalool, anethole, and estragole had a higher correlation coefficient $(0.79 \leq \mathrm{r} \leq 0.99)$ with $\mathrm{SP}_{3}$ and a lower correlation coefficient $(-0.15 \leq \mathrm{r} \leq 0.21)$ with $\mathrm{SP}_{4}, \mathrm{SP}_{5}$, and $\mathrm{SP}_{6}$, this suggests that they may be potential flavour markers to distinguish $\mathrm{SP}_{3}$ and $\mathrm{SP}_{4}, \mathrm{SP}_{5}$ and $\mathrm{SP}_{6}$.

(a)

\section{Correlations on axes t 1 and $\mathbf{t} 2$}

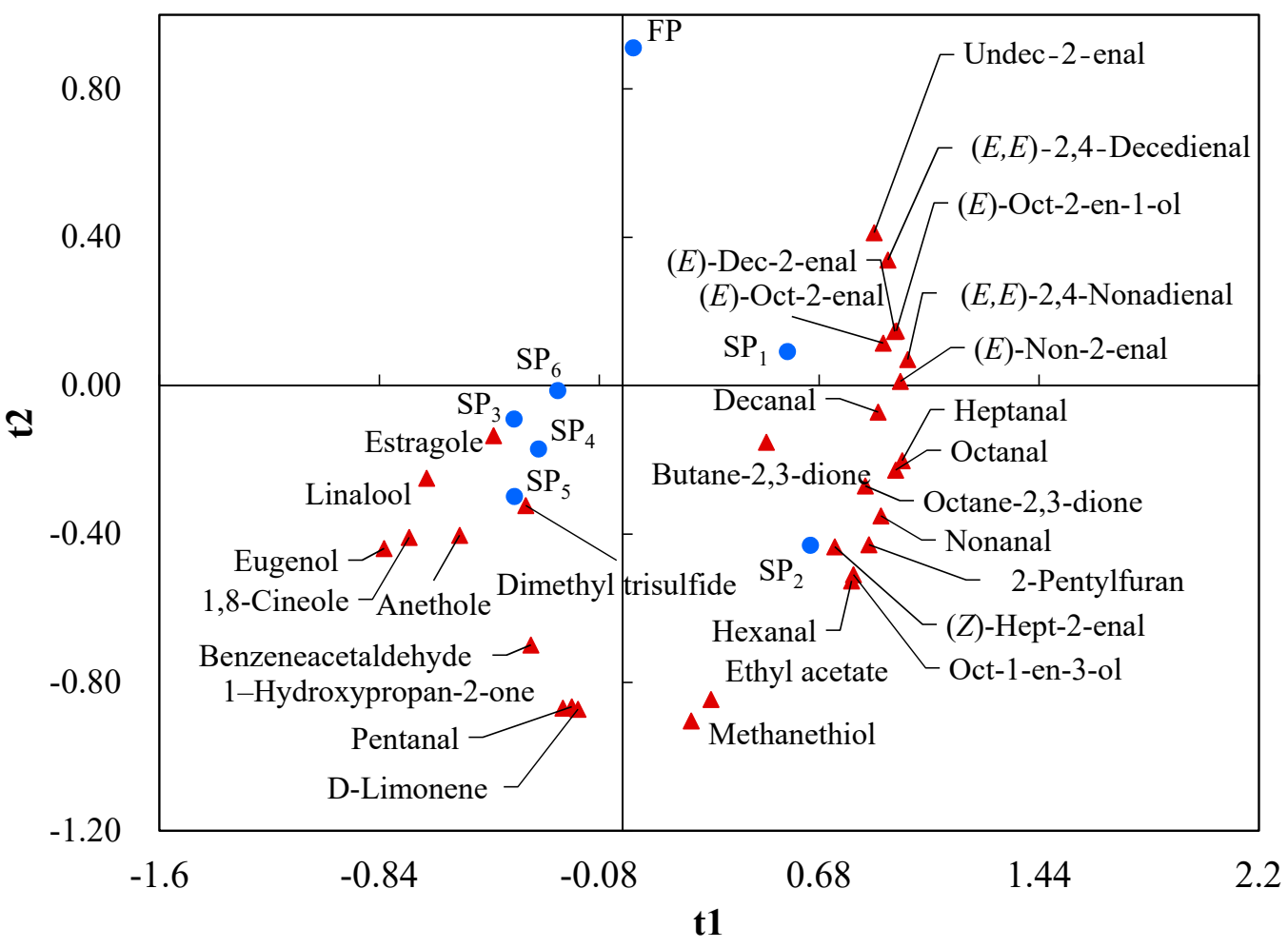

Figure 3. Cont. 
(b)

\begin{tabular}{|c|c|c|c|c|c|c|c|}
\hline (b) & 宝 & 感 & 光 & $\hat{\sim}$ & $\vec{n}$ & $\stackrel{n}{n}$ & $\hat{\infty}^{\circ}$ \\
\hline Pentanal & -0.83 & -0.20 & 0.30 & 0.46 & -0.03 & 0.20 & 0.10 \\
\hline Hexanal & -0.56 & 0.60 & 0.60 & -0.14 & -0.10 & -0.24 & -0.16 \\
\hline Heptanal & -0.11 & 0.46 & 0.78 & -0.32 & -0.28 & -0.26 & -0.27 \\
\hline Octanal & -0.14 & 0.46 & 0.76 & -0.28 & -0.13 & -0.35 & -0.32 \\
\hline (Z)-Hept-2-enal & -0.19 & -0.02 & 0.97 & -0.19 & -0.19 & -0.19 & -0.19 \\
\hline Nonanal & -0.24 & 0.31 & 0.87 & -0.15 & -0.28 & -0.33 & -0.18 \\
\hline (E)-Oct-2-enal & 0.01 & 0.85 & 0.30 & -0.34 & -0.30 & -0.28 & -0.25 \\
\hline Decanal & -0.15 & 0.69 & 0.45 & -0.32 & -0.30 & -0.37 & 0.00 \\
\hline (E)-Non-2-enal & 0.12 & 0.42 & 0.73 & -0.34 & -0.33 & -0.28 & -0.32 \\
\hline Benzeneacetaldehyde & -0.57 & -0.25 & 0.04 & -0.34 & 0.31 & 0.66 & 0.15 \\
\hline (E)-Dec-2-enal & 0.13 & 0.67 & 0.48 & -0.28 & -0.36 & -0.33 & -0.31 \\
\hline$(E, E)-2,4$-Nonadienal & 0.10 & 0.62 & 0.58 & -0.33 & -0.33 & -0.33 & -0.33 \\
\hline Undec-2-enal & 0.46 & 0.51 & 0.41 & -0.35 & -0.35 & -0.35 & -0.35 \\
\hline$(E, E)-2,4$-Decedienal & 0.34 & 0.62 & 0.40 & -0.35 & -0.43 & -0.34 & -0.26 \\
\hline
\end{tabular}

Figure 3. Cont. 


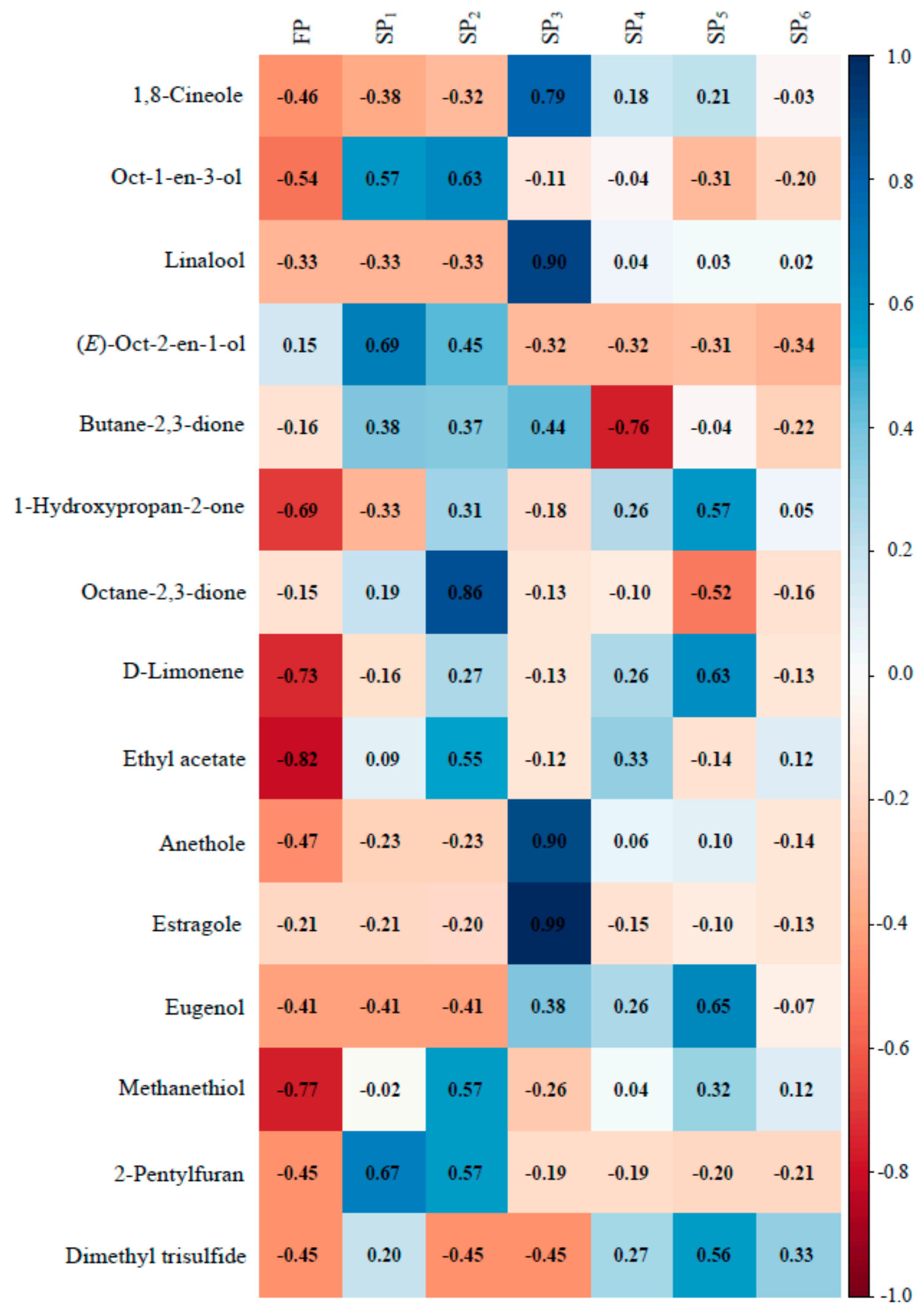

Figure 3. (a) Loading biplot of $\mathrm{t} 1$ and $\mathrm{t} 2$ of the model performed after PLS-DA of the volatile compounds in different pork samples $\left(R^{2} X=0.968, R^{2} Y=0.818\right.$ and $\left.Q^{2}=0.628\right)$. (b) Heat map of the correlations between volatile compounds and the pork samples. FP, fresh pork; $\mathrm{SP}_{1}$, stewed pork with water; $\mathrm{SP}_{2}$ : stewed pork with water and salt; $\mathrm{SP}_{3}$ : stewed pork with water, salt and spices; $\mathrm{SP}_{4}$ : stewed pork with water, salt, spices and soy sauce; $\mathrm{SP}_{5}$ : stewed pork with water, salt, spices, soy sauce and sugar; $\mathrm{SP}_{6}$ : stewed pork with water, salt, spices, soy sauce, sugar and cooking wine. The blue circle dots represent the fresh and stewed pork and the red triangle dots represent the odour-active compounds. 


\subsection{Descriptive Sensory Analysis}

To describe the differences of odour profiles of seven pork samples, the flavour sensory evaluation was performed using the five representative descriptors, namely, "meaty", "spicy", "caramel", "soy sauce" and "fatty". As can be seen from Figure 4. Significant differences ( $p \leq 0.01$ ) were found among five odour attributes of the pork samples. The intensities of fatty notes in $\mathrm{SP}_{1}$ and $\mathrm{SP}_{2}$ were highest, followed by $\mathrm{SP}_{3}, \mathrm{SP}_{4}, \mathrm{SP}_{5}, \mathrm{SP}_{6}$ and $\mathrm{FP}$. As described by the panellists from GC-MS/O, hexanal, heptanal, octanal, (Z)-hept-2-enal, nonanal, $(E)$-oct-2-enal, decanal, $(E)$-non-2-enal, $(E)$-dec-2-enal, $(E, E)$-2,4-nonadienal, $(E, E)$ 2,4-decedienal and 2-pentylfuran might be closely related to the fatty odour. This result agreed with the result of Figure $3 \mathrm{~b}$. The meaty and caramel odour of $\mathrm{SP}_{5}$ and $\mathrm{SP}_{6}$ had the highest score in all samples, which could be mainly attributed to furans and $\mathrm{N}$-containing compounds such as 3-(4-methyl-3-pentenyl)-furan, furfural, 2-furanmethanol, pyridine, and 2-acetylpryrrole. In addition, the strong spicy and soy sauce smell was presented in $\mathrm{SP}_{3}$ and $\mathrm{SP}_{5}$, respectively.

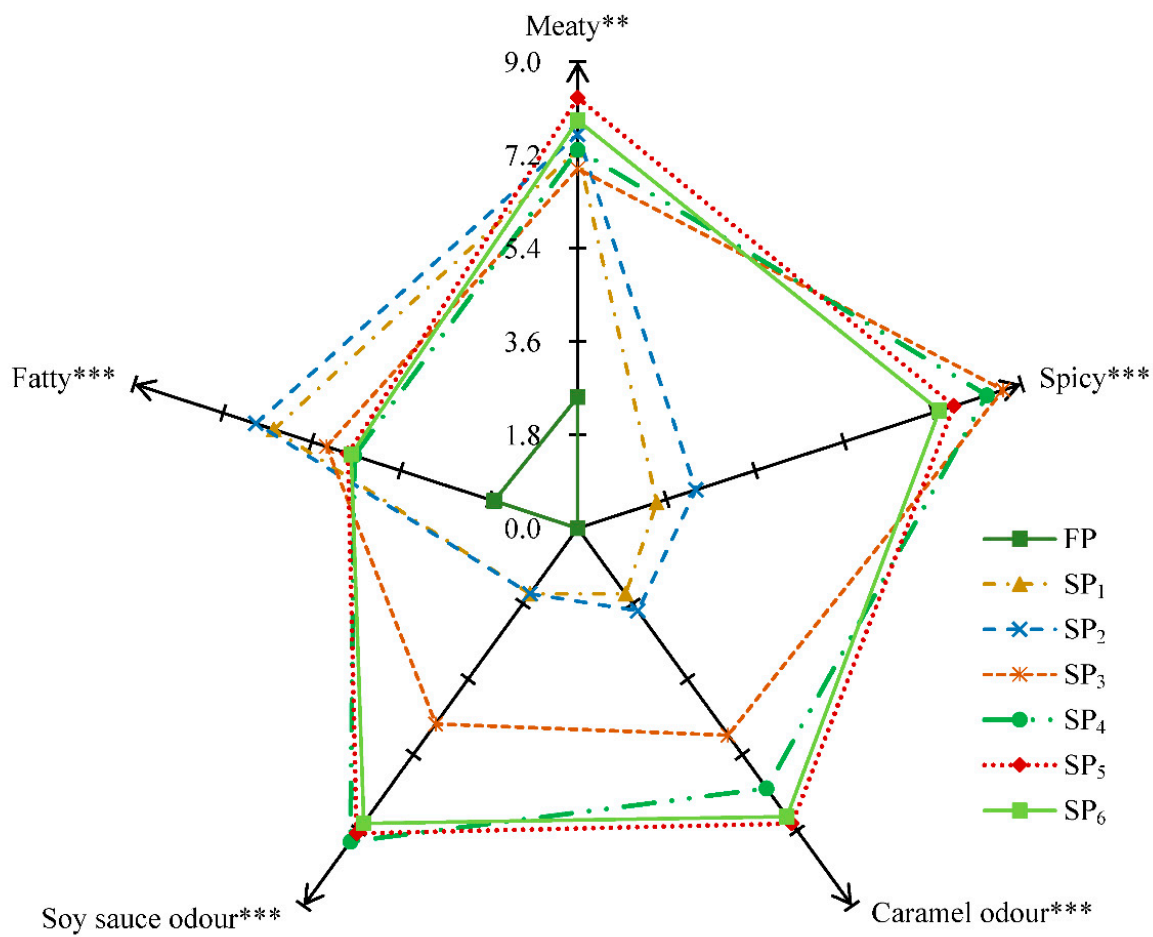

Figure 4. The odour sensory profiles of the fresh and stewed pork. Asterisks indicate significant $\left.{ }^{* *} p \leq 0.01{ }^{* * *} p \leq 0.001\right)$ differences of means in descriptor intensities. FP, fresh pork; SP1, stewed pork with water; SP2: stewed pork in water and salt; SP3: stewed pork in water, salt and spices; SP4: stewed pork in water, salt, spices and soy sauce; SP5: stewed pork in water, salt, spices, soy sauce and sugar; SP6: stewed pork in water, salt, spices, soy sauce, sugar and cooking wine.

\subsection{Relationship between Sensory Evaluation and Odour-Active Compounds}

PLSR was employed to establish the relationship between the five sensory descriptors of the fresh and stewed pork and the odour-active compounds analysed by GC-MS/O and GC $\times$ GC-TOFMS, and the correlation coefficient between them was expressed in the heat map. As shown in Figure 5a, most of the $X$-matrix (contribution ratios of the odour-active compounds) and $Y$-matrix (intensities of the sensory attributes) are loaded around the circle $\left(r^{2}=100 \%, r^{2}=\right.$ represent the degree of interpretation). The model quality $\left(Q^{2}=0.846\right) \geq 0.50$ indicated that they were well explained by the PLSR model. The first two components explained $74.0 \%$ of $X$-matrix and $92.7 \%$ of $Y$-matrix. The dots corresponding to sample $\mathrm{SP}_{1}$ and $\mathrm{SP}_{2}$ had overlap in the second quadrant, and the samples points of $\mathrm{SP}_{3}, \mathrm{SP}_{4}, \mathrm{SP}_{5}$ and $\mathrm{SP}_{6}$ were close in the fourth quadrant, and as well as $\mathrm{FP}$ was 
found in the third quadrant. So, the fresh and stewed pork samples can be divided into three group and this result was consistent with previous PCA plots (Figure 2a). According to Figure $5 \mathrm{a}, \mathrm{b}$, it can be observed that $\mathrm{SP}_{3}, \mathrm{SP}_{4}, \mathrm{SP}_{5}$, and $\mathrm{SP}_{6}$ were characterized by soy sauce, caramel and spicy odour because of their short distance, and the three aroma attributes aforementioned were positively correlated with pentanal, benzeneacetaldehyde, 1,8-cineole, 1-hydroxypropan-2-one, D-limonene, ethyl acetate, eugenol, methanethiol, and dimethyl trisulfide with a high correlation coefficient $(0.60 \leq \mathrm{r} \leq 0.92)$. On the contrary, the soy sauce, caramel and spicy notes located in the right side of the loading plot were strongly and negatively correlated with some aldehydes (heptanal, nonanal, $(E)$-oct-2-enal, (E)-non-2-enal, $(E)$-dec-2-enal, $(E, E)$-2,4-nonadienal, undec-2-enal and $(E, E)$-2,4-nonadienal) and unsaturated alcohols like (E)-oct-2-en-1-ol. $\mathrm{SP}_{1}$ and $\mathrm{SP}_{2}$ on the upper left side of loading plot were mainly descriptive fatty and meaty odour, which were in accordance with the descriptive sensory analysis. These two attributes were highly associated with hexanal, oct-1-en-3-ol and 2-pentylfuruan. Moreover, FP was located far from these flavour attributions and most volatiles, which indicated that there was not the unique flavour of the fresh pork.

\section{(a)}

\section{Correlations on axes $\mathrm{t} 1$ and $\mathrm{t} 2$}

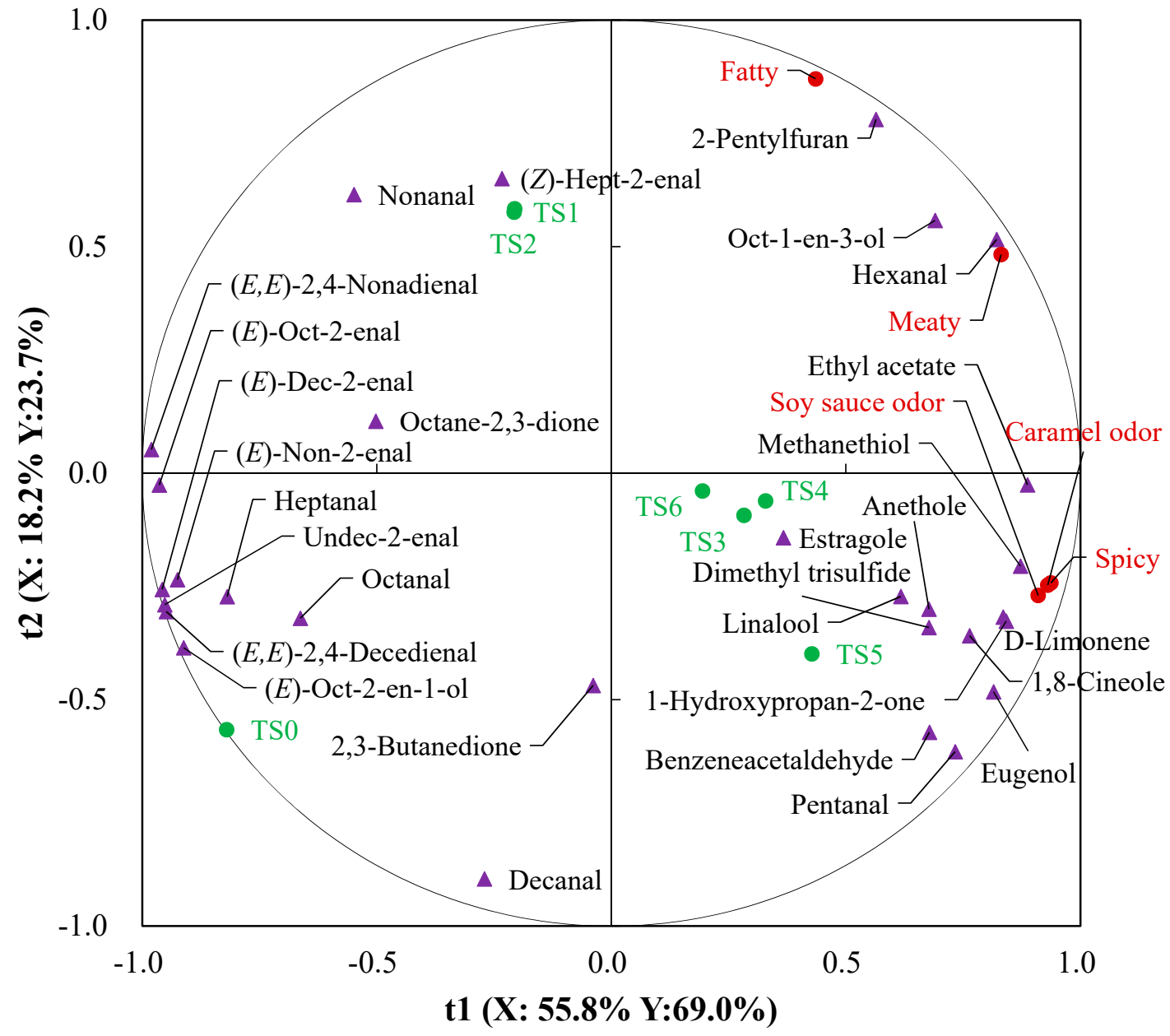

Figure 5. Cont. 


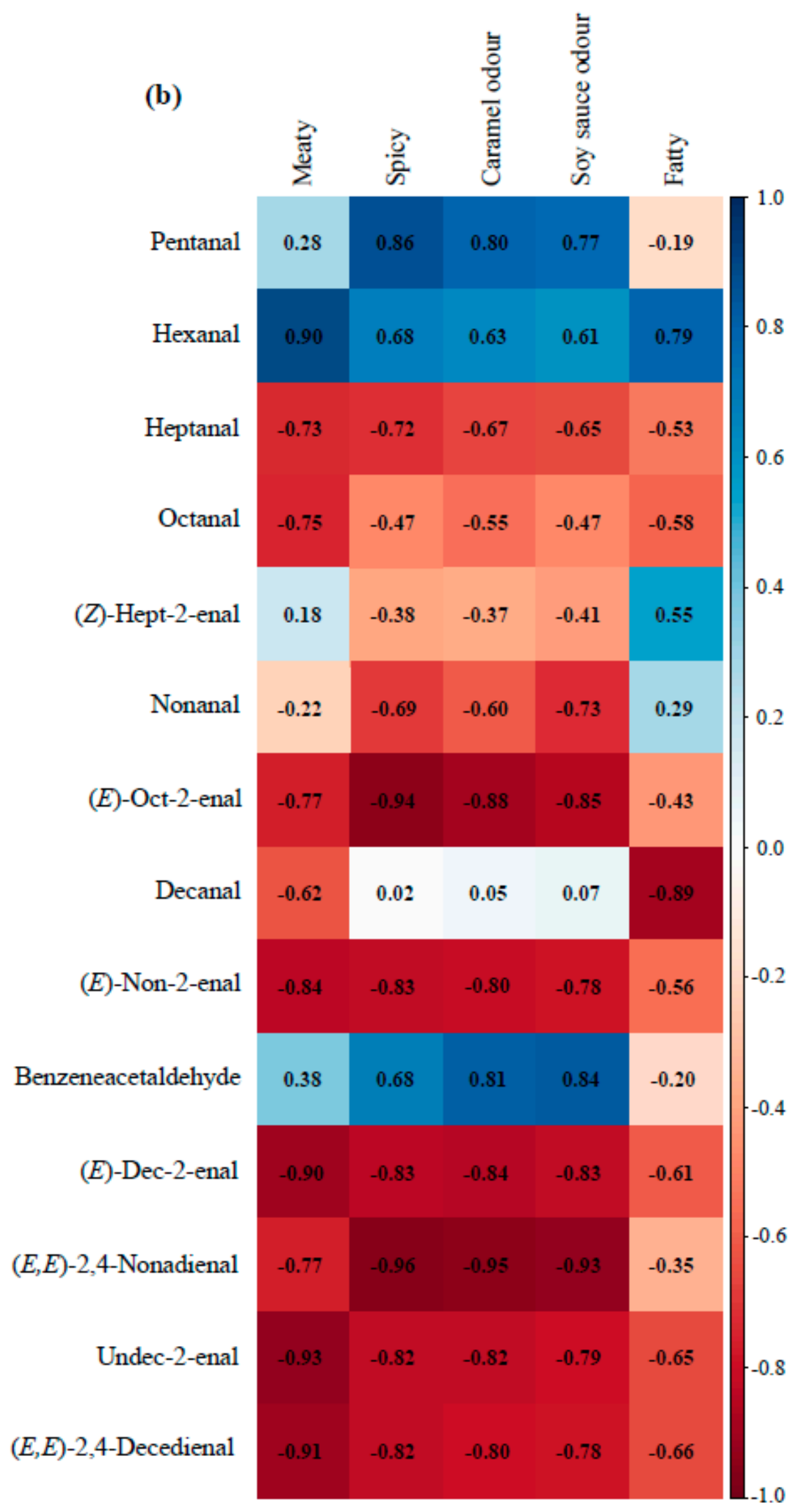

Figure 5. Cont. 


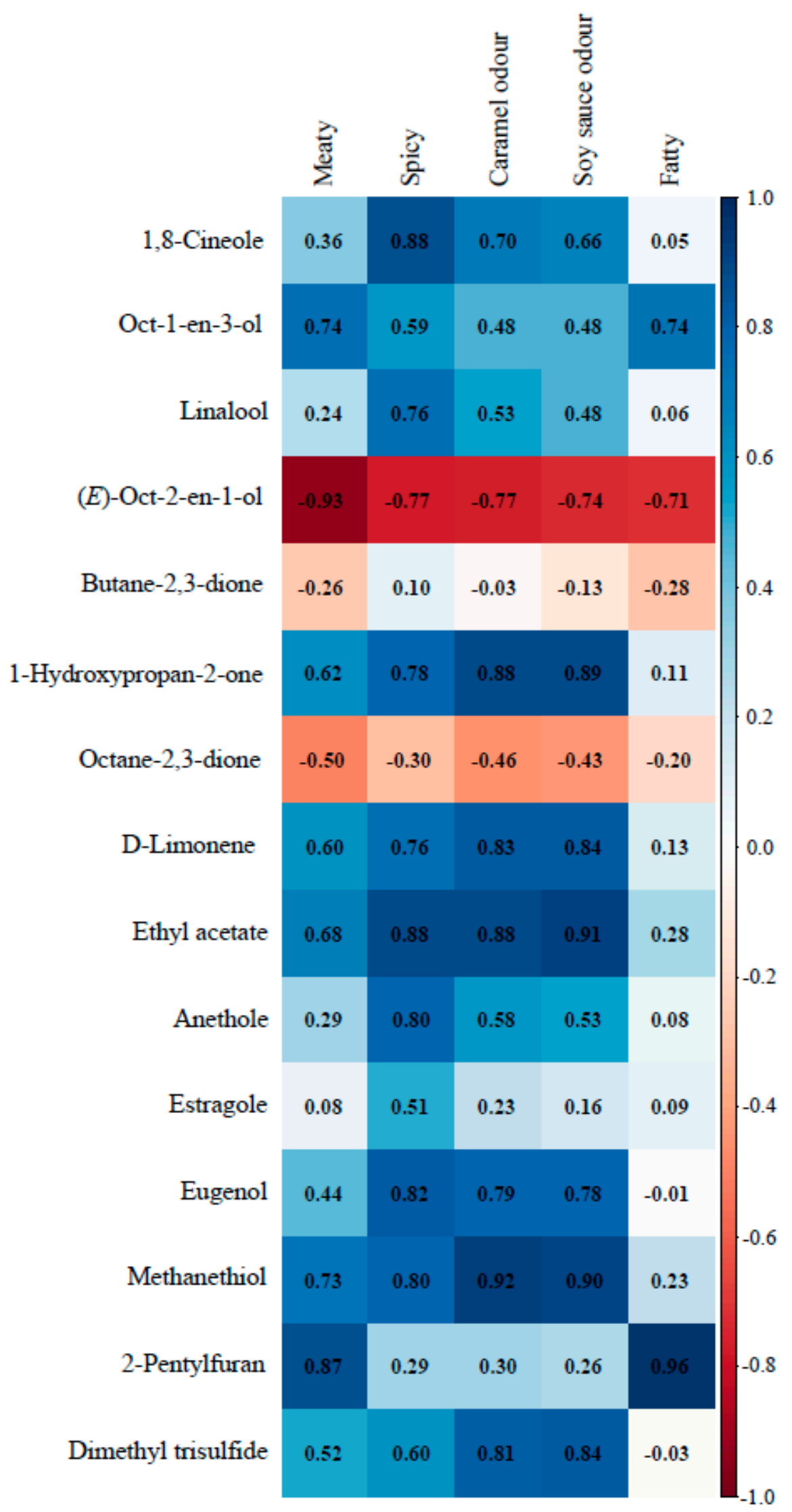

Figure 5. (a) PLSR loading for the odour attributes ( $Y$ variables) and the odour-active compounds of the fresh and stewed pork (X variables). (b) Heat map illustrating the Pearson correlation between descriptor intensities and proportion of OAVs of the odouractive compounds calculated by PLSR. The green circle dots represent 7 pork samples, the red circle dots represent 5 aroma attributes and the purple triangle dots represent 29 odour-active compounds. 


\section{Conclusions}

The volatile profile of fresh and stewed pork was more fully characterized using GC-MS/O and GC $\times$ GC-TOFMS analysis. A total of 139 volatile compounds were identified from all stewed pork and seven of which were confirmed as the key odour-active compounds, namely hexanal, heptanal, octanal, nonanal, oct-1-en-3-ol, 2-pentylfuran, and methanethiol. The fresh and stewed pork could be classified to four groups ( $\mathrm{FP}, \mathrm{SP}_{1}$ $\mathrm{SP}_{2}, \mathrm{SP}_{3}$, and $\mathrm{SP}_{4}-\mathrm{SP}_{5}-\mathrm{SP}_{6}$ ) by PCA based on the odour-active compounds, which showed that volatile profile of pork stewed with water and salt possessed the similar flavour, and the flavour composition of stewed pork with soy sauce, sugar, and cooking wine was not significantly different. However, there were significant differences in the overall flavour between pork samples of different groups. It can be concluded that the seasoning played a vital role in the flavour contribution of stewed pork, especially salt and spices. Additionally, the results of PLSR indicated that samples $\mathrm{SP}_{3}, \mathrm{SP}_{4}, \mathrm{SP}_{5}$, and $\mathrm{SP}_{6}$ were positively correlated with soy sauce, caramel, and spicy notes. On the contrary, soy sauce, caramel, and spicy odours were strongly and negatively correlated with samples $\mathrm{SP}_{1}$ and $\mathrm{SP}_{2}$, which was consistent with the result of sensory evaluation.

Supplementary Materials: The following are available online at https:/ / www.mdpi.com/2304-815 8/10/1/83/s1, Figure S1: Flow diagram of the stewed pork. Table S1: Information of the definitions and reference standards of odour attributes.

Author Contributions: Formal analysis and writing —original draft, D.H.; funding acquisition and methodology, C.-H.Z.; writing-review and editing, M.-L.F. All authors have read and agreed to the published version of the manuscript.

Funding: This research was funded by "N Special Project" of Institute of Food Science and Technology, Chinese Academy of Agricultural Sciences (SN2020-01) and Central Public-interest Scientific Institution Basal Research Fund (Y2020CG08).

Institutional Review Board Statement: This article does not contain any studies with human participants performed by any of the authors.

Informed Consent Statement: Informed consent was obtained from all individual participants includedin the study.

Data Availability Statement: The data presented in this study are available in the article and supplementary material.

Conflicts of Interest: All authors declare that they have no conflict of interest.

\section{References}

1. Yang, Y.; Pan, D.; Sun, Y.; Wang, Y.; Xu, F.; Cao, J. ${ }^{1} \mathrm{H}$ NMR-based metabolomics profiling and taste of stewed pork-hock in soy sauce. Food Res. Int. 2019, 121, 658-665. [CrossRef]

2. Han, D.; Mi, S.; Zhang, C.H.; Li, J.; Song, H.L.; Fauconnier, M.L.; Tyteca, E. Characterization and discrimination of Chinese marinated pork hocks by volatile compound profiling using solid phase microextraction gas chromatography-mass spectrometry/olfactometry, electronic nose and chemometrics. Molecules 2019, 24, 1385. [CrossRef]

3. Zeng, W.; Wen, W.; Deng, Y.; Tian, Y.; Sun, H.; Sun, Q. Chinese ethnic meat product: Continuity and development. Meat Sci. 2016, 120, 37-46. [CrossRef]

4. Qin, Y.X.; Cai, D.D.; Zhang, D.N.; Liu, Y.; Lai, K.Q. Characteristics of volatile flavor component in stewed meat and meat broths prepared with repeatedly used broths containing star anise. J. Food Meas. Charact. 2019, 14, 557-572. [CrossRef]

5. Liu, T.T.; Yang, T.S.; Wu, C.M. Changes of volatiles in soy sauce-stewed pork during cold storage and reheating. J. Sci. Food Agric. 2001, 81, 1547-1552. [CrossRef]

6. Overholt, M.F.; Mancini, S.; Galloway, H.O.; Preziuso, G.; Dilger, A.C.; Boler, D.D. Effect of salt purity on lipid oxidation, sensory characteristics, and textural properties of fresh, ground pork patties. LWT Food Sci. Technol. 2016, 65, 890-896. [CrossRef]

7. Mariutti, L.R.; Bragagnolo, N. Influence of salt on lipid oxidation in meat and seafood product: A review. Food Res. Int. 2017, 94, 90-100. [CrossRef]

8. Feng, X.; Ahn, D.U. Volatile profile, lipid oxidation and protein oxidation of irradiated ready-to-eat cured turkey meat product. Radiat. Phys. Chem. 2016, 127, 27-33. [CrossRef]

9. Wang, P.; Hong, Y.; Ke, W.; Hu, X.; Chen, F. Formation of heterocyclic amines in Chinese marinated meat: Effect of animal species and ingredient (rock candy, soy sauce and rice wine). J. Sci. Food Agric. 2017, 97, 3967-3978. [CrossRef] 
10. Duan, Y.; Zheng, F.; Chen, H.; Huang, M.; Xie, J.; Chen, F.; Sun, B. Analysis of volatiles in Dezhou Braised Chicken by comprehensive two-dimensional gas chromatography/high resolution-time of flight mass spectrometry. LWT Food Sci. Technol. 2015, 60, 1235-1242. [CrossRef]

11. Olivares, A.; Navarro, J.L.; Flores, M. Effect of fat content on aroma generation during processing of dry fermented sausages. Meat Sci. 2011, 87, 264-273. [CrossRef] [PubMed]

12. Lu, F.; Kuhnle, G.K.; Cheng, Q. The effect of common spices and meat type on the formation of heterocyclic amines and polycyclic aromatic hydrocarbons in deep-fried meatballs. Food Control. 2018, 92, 399-411. [CrossRef]

13. Yang, H.S.; Lee, E.J.; Moon, S.H.; Paik, H.D.; Ahn, D.U. Addition of garlic or onion before irradiation on lipid oxidation, volatiles and sensory characteristics of cooked ground beef. Meat Sci. 2011, 88, 286-291. [CrossRef] [PubMed]

14. Sun, L.; Chen, J.; Li, M.; Liu, Y.; Zhao, G. Effect of star anise (Illicium verum) on the volatile compounds of stewed chicken. J. Food Process. Eng. 2014, 37, 131-145. [CrossRef]

15. Zhao, J.; Wang, M.; Xie, J.; Zhao, M.; Hou, L.; Liang, J.J.; Wang, S.; Cheng, J. Volatile flavor constituent in the pork broth of black-pig. Food Chem. 2017, 226, 51-60. [CrossRef] [PubMed]

16. Song, S.; Fan, L.; Xu, X.; Xu, R.; Jia, Q.; Feng, T. Aroma Patterns Characterization of braised pork obtained from a novel ingredient by sensory-guided analysis and gas-chromatography-olfactometry. Foods 2019, 8, 87. [CrossRef]

17. Huang, X.H.; Zheng, X.; Chen, Z.H.; Zhang, Y.Y.; Du, M.; Dong, X.P.; Qin, D.L.; Zhu, B.W. Fresh and grilled eel volatile fingerprinting by E-nose, GC-O, GC-MS and GC $\times$ GC-QTOF combined with purge and trap and solvent-assisted flavor evaporation. Food Res. Int. 2019, 115, 32-43. [CrossRef]

18. Wang, W.; Feng, X.; Zhang, D.; Li, B.; Sun, B.; Tian, H.; Liu, Y. Analysis of volatile compounds in Chinese dry-cured hams by comprehensive two-dimensional gas chromatography with high-resolution time-of-flight mass spectrometry. Meat Sci. 2018, 140, 14-25. [CrossRef]

19. Zhu, Y.; Lv, H.P.; Shao, C.Y.; Kang, S.; Zhang, Y.; Guo, L.; Dai, W.D.; Tan, J.F.; Peng, Q.H.; Lin, Z. Identification of key odorant responsible for chestnut-like aroma quality of green teas. Food Res. Int. 2018, 108, 74-82. [CrossRef]

20. Li, H.; Li, X.; Zhang, C.H.; Wang, J.Z.; Tang, C.H.; Chen, L.L. Flavor compounds and sensory profiles of a novel Chinese marinated chicken. J. Sci. Food Agric. 2016, 96, 1618-1626. [CrossRef]

21. Han, D.; Zhang, C.H.; Fauconnier, M.L.; Mi, S. Characterization and differentiation of boiled pork from Tibetan, Sanmenxia and Duroc $\times($ Landrace $\times$ Yorkshire $)$ pigs by volatiles profiling and chemometrics analysis. Food Res. Int. 2019, 130, 108910. [CrossRef] [PubMed]

22. Wang, Y.; Song, H.; Zhang, Y.; Tang, J.; Yu, D. Determination of aroma compounds in pork broth produced by different processing methods. Flavour Fragr. J. 2016, 31, 319-328. [CrossRef]

23. Aaslyng, M.D.; Meinert, L. Meat flavour in pork and beef-from animal to meal. Meat Sci. 2017, 132, 112-117. [CrossRef] [PubMed]

24. Yang, Y.; Sun, Y.; Pan, D.; Wang, Y.; Cao, J. Effect of high pressure treatment on lipolysis-oxidation and volatiles of marinated pork meat in soy sauce. Meat Sci. 2018, 145, 186-194. [CrossRef]

25. Lorenzo, J.M.; Fonseca, S. Volatile compounds of Celta dry-cured 'lacon' as affected by cross-breeding with Duroc and Landrace genotypes. J. Sci. Food Agric. 2014, 94, 2978-2985. [CrossRef] [PubMed]

26. Zou, Y.; Kang, D.; Liu, R.; Qi, J.; Zhou, G.; Zhang, W. Effect of ultrasonic assisted cooking on the chemical profiles of taste and flavor of spiced beef. Ultrason. Sonochem. 2018, 46, 36-45. [CrossRef]

27. Petričević, S.; Radovčić, N.M.; Lukić, K.; Listeš, E.; Medić, H. Differentiation of dry-cured hams from different processing methods by means of volatile compounds, physico-chemical and sensory analysis. Meat Sci. 2018, 137, 217-227. [CrossRef]

28. Jayasena, D.D.; Ahn, D.U.; Nam, K.C.; Jo, C. Flavour chemistry of chicken meat: A review. Asian Australas. J. Anim. Sci. 2013, 26, 732-742. [CrossRef]

29. Gu, S.Q.; Wang, X.C.; Tao, N.P.; Wu, N. Characterization of volatile compounds in different edible part of steamed Chinese mitten crab (Eriocheir sinensis). Food Res. Int. 2013, 54, 81-92. [CrossRef]

30. Gong, H.; Yang, Z.; Liu, M.; Shi, Z.; Li, J.; Chen, W.; Qiao, X. Time-dependent categorization of volatile aroma compound formation in stewed Chinese spicy beef using electron nose profile coupled with thermal desorption GC-MS detection. Food Sci. Hum. Wellness 2017, 6, 137-146. [CrossRef]

31. Toldrá, F. Lawrie's Meat Science, 8th ed.; Woodhead Publishing: Cambridge, UK, 2017; pp. $383-417$.

32. Van Ba, H.; Hwang, I.; Jeong, D.; Touseef, A. Principle of meat aroma flavors and future prospect. Latest Res. Qual. Control. 2012, 2, 145-176.

33. Pham, A.; Schilling, M.; Mikel, W.; Williams, J.; Martin, J.; Coggins, P. Relationships between sensory descriptors, consumer acceptability and volatile flavor compounds of American dry-cured ham. Meat Sci. 2008, 80, 728-737. [CrossRef] [PubMed]

34. Lorenzo, J.M.; Montes, R.; Purriños, L.; Franco, D. Effect of pork fat addition on the volatile compounds of foal dry-cured sausage. Meat Sci. 2012, 91, 506-512. [CrossRef] [PubMed]

35. Qi, J.; Liu, D.Y.; Zhou, G.H.; Xu, X.L. Characteristic flavor of traditional soup made by stewing Chinese yellow-feather chickens. J. Food Sci. 2017, 82, 2031-2040. [CrossRef] [PubMed]

36. Ramírez, R.; Cava, R. Volatile profiles of dry-cured meat product from three different Iberian $\times$ Duroc genotypes. J. Agric. Food Chem. 2007, 55, 1923-1931. [CrossRef] [PubMed]

37. Shahidi, F. Flavor of Meat, Meat Product and Seafood, 2nd ed.; Chapman and Hall Publishing Group: London, UK, $1998 ;$ pp. 5-23. 
38. Lorenzo, J.M.; Franco, D.; Carballo, J. Effect of the inclusion of chestnut in the finishing diet on volatile compounds during the manufacture of dry-cured "Lacón" from Celta pig breed. Meat Sci. 2014, 96, 211-223. [CrossRef] [PubMed]

39. Liu, Y.; Xu, X.L.; Ouyang, G.F.; Zhou, G.H. Changes in volatile compounds of traditional Chinese Nanjing water-boiled salted duck during processing. J. Food Sci. 2006, 71, S371-S377. [CrossRef]

40. Tai, C.Y.; Ho, C.T. Influence of cysteine oxidation on thermal formation of Maillard aromas. J. Agric. Food Chem. 1997, 45, 3586-3589. [CrossRef]

41. Zhou, X.; Chong, Y.; Ding, Y.; Gu, S.; Liu, L. Determination of the effect of different washing processes on aroma characteristics in silver carp mince by MMSE-GC-MS, e-nose and sensory evaluation. Food Chem. 2016, 207, 205-213. [CrossRef]

42. Lotfy, S.N.; Fadel, H.H.; El-Ghorab, A.H.; Shaheen, M.S. Stability of encapsulated beef-like flavourings prepared from enzymatically hydrolysed mushroom proteins with other precursors under conventional and microwave heating. Food Chem. 2015, 187, 7-13. [CrossRef] 